\title{
Simulation of atomic zinc luminescence in rare gas solids: A localized pair potentials approach
}

\author{
John G. McCaffrey and Paul N. Kerins \\ Department of Chemistry, St. Patrick's College, Maynooth, County Kildare, Ireland
}

(Received 2 October 1996; accepted 12 February 1997)

\begin{abstract}
The luminescence spectroscopy of atomic zinc isolated in the solid rare gases (Zn/RG) is compared with theoretical predictions obtained from the sum of diatomic $\mathrm{Zn} \cdot \mathrm{RG}$ and $\mathrm{RG} \cdot \mathrm{RG}$ pair potentials. In particular the existence of pairs of emission bands, both of which are assigned to the same gas phase electronic transition, is examined with the use of diatomic pair potentials to simulate the potential energy surfaces of the Jahn-Teller active vibrational modes of $\mathrm{Zn}$ in the solid rare gases $\mathrm{Ar}, \mathrm{Kr}$, and Xe. Simulations of the solid state Zn/RG luminescence are developed from a consideration of the excited state $\mathrm{Zn}\left({ }^{1} P_{1}\right) \cdot \mathrm{RG}_{n}$ van der Waals cluster species in the gas phase. The maximum binding energy of the $\mathrm{Zn}\left({ }^{1} P_{1}\right) \cdot \mathrm{RG}_{n}$ clusters is found in the $\mathrm{Zn} \cdot \mathrm{RG}_{4}$ cluster having a square planar structure at the energy minimum. Based on the results of the cluster calculations, lattice distortions which led to a dominant interaction between the $\mathrm{Zn}$ atom and four of its host atoms were sought to simulate the solid state luminescence. Two such vibronic modes were identified; one a lattice mode in which four rare gas atoms contract on a single plane toward the $\mathrm{Zn}$ atom, referred to as the waist mode, and the other a motion of the $\mathrm{Zn}$ atom toward an octahedral interstitial site of the lattice, the body mode. Energy calculations of these modes were carried out for rigid and relaxed rare gas lattices allowing identification of the high energy emission bands in the Zn/RG systems as arising from the waist mode, while the lower energy bands are associated with the body mode. The model also rationalizes the differences exhibited in the time-resolved behavior of the pairs of singlet emission bands in the $\mathrm{Zn} / \mathrm{Ar}$ and $\mathrm{Zn} / \mathrm{Kr}$ systems, whereby the lower energy band of a given system shows a risetime of a few hundred picoseconds while the higher energy band exhibits direct feeding. The steep gradient calculated on the waist mode, feeding the high energy band, and the flat gradient found on the body mode, feeding the lower energy emission, are consistent with the existence of a risetime in the latter and its absence in the former. The close agreement found between theory and experiment indicates the validity of using pair potentials in analysis of matrix zinc spectroscopy and thereby indicates that the luminescence is controlled by localized guest-host interactions. (C) 1997 American Institute of Physics.
\end{abstract}

[S0021-9606(97)01319-6]

\section{INTRODUCTION}

The relative importance of localized versus delocalized vibronic modes in determining the band shapes of electronic transitions of optically active centres in solids has long been sought. Previous attempts have been hampered either by complex packing structures or lack of accurate guest-host interaction potentials. Studies of metal atoms isolated in the solid rare gases ${ }^{1}$ offer a solution because (a) the packing structures of the solid rare gases are particularly simple being face centered cubic (fcc) and (b) highly accurate potentials are now increasingly available from spectroscopic data for the diatomic metal atom/rare gas atom van der Waals molecules $(\mathrm{M} \cdot \mathrm{RG})$. In this contribution the luminescence spectroscopy of atomic zinc isolated in the solid rare gases ( $\mathrm{Zn} /$ $\mathrm{RG}$ ) is compared with theoretical predictions based on the sum of pair potentials since the diatomic $\mathrm{Zn} \cdot \mathrm{RG}$ potentials ${ }^{2}$ are now available.

Details of the luminescence recorded in the $\mathrm{Zn} / \mathrm{RG}$ systems have been presented elsewhere ${ }^{3}$ but a summary of the features relevant to the spectral simulations follows. Samples of atomic zinc isolated in thin films $(10-30 \mu \mathrm{m})$ of the solid rare gases $\mathrm{Zn} / \mathrm{RG}$ were prepared by the codeposition of atomic $\mathrm{Zn}$ vapor, generated by electron bombardment of a 1-mm-diam zinc wire, with the rare gases. Luminescence spectroscopy was recorded at the HIGITI beamline of the synchrotron radiation facility HASYLAB at DESY, Hamburg. Excited state lifetime measurements were made using the time correlated single photon counting technique.

Figure 1 shows a summary of the luminescence spectroscopy ${ }^{4}$ recorded for atomic zinc isolated in solid $\mathrm{Ar}$, $\mathrm{Kr}$, and Xe while Table I gives the spectral positions and measured lifetimes of the bands shown in the figure. It is immediately evident on the left side of Fig. 1 that all the excitation profiles show a threefold splitting behavior previously ${ }^{5}$ attributed to dynamic Jahn-Teller coupling in the excited ${ }^{1} T_{1 u}$ electronic state. As described elsewhere, ${ }^{3}$ careful annealing studies ${ }^{6}$ indicate that all the emission bands shown in Fig. 1 are associated with the occupancy of atomic zinc in the most thermally stable site of the solid rare gases. For comparison the positions of the singlet $\left(4 s 4 p{ }^{1} P_{1}\right.$ $\left.\leftrightarrow 4 s^{2}{ }^{1} S_{0}\right)$ and triplet $\left(4 s 4 p{ }^{3} P_{1} \leftrightarrow 4 s^{2}{ }^{1} S_{0}\right)$ resonance transitions of atomic zinc in the gas phase are also presented in Fig. 1. From the recorded spectral positions and emission lifetimes, the pair of emission bands observed in the UV in 


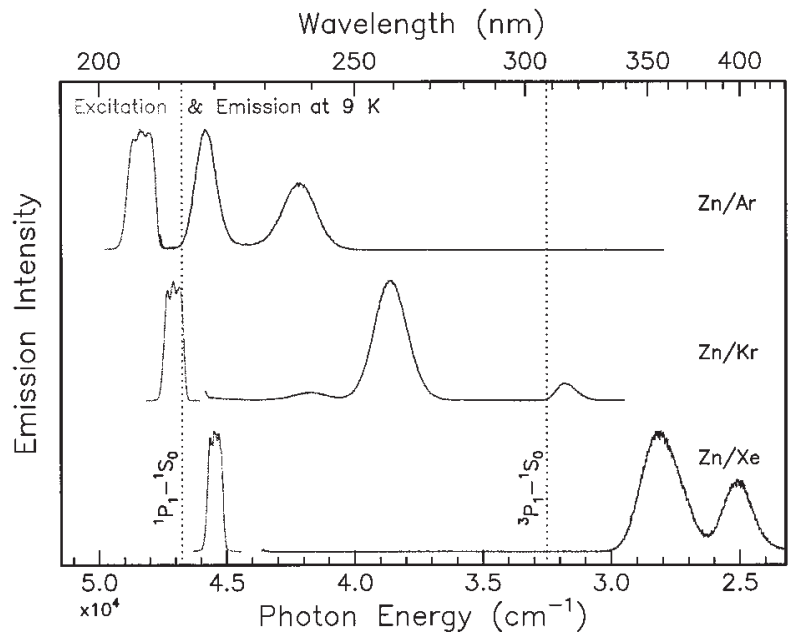

FIG. 1. Excitation and emission profiles recorded at $9 \mathrm{~K}$ in the annealed $\mathrm{Zn} / \mathrm{RG}$ matrix systems. For a given Zn/RG system, the excitation profile shown on the left is recorded by monitoring any of the emission bands shown on the right. The excitation wavelengths used in producing the emission spectra were 207, 212, and $220 \mathrm{~nm}$ for $\mathrm{Zn} / \mathrm{Ar}, \mathrm{Zn} / \mathrm{Kr}$, and $\mathrm{Zn} / \mathrm{Xe}$, respectively. The spectral positions of the resonance singlet $4 s 4 p^{1} P_{1}$ $-4 s^{2}{ }^{1} S_{0}$ and triplet $4 s 4 p^{3} P_{1}-4 s^{2}{ }^{1} S_{0}$ transitions of atomic zinc in the gas phase are shown by the dotted vertical lines for comparison.

Ar and Kr matrices are assigned to the singlet transition, while the bands in the near $\mathrm{UV}$ in $\mathrm{Kr}$ and $\mathrm{Xe}$ are assigned as the triplet emission of atomic zinc.

In the present work, the reason for the existence of pairs of emission bands in the Zn/RG solids, where a only single transition of atomic zinc occurs in the gas phase, is examined with the use of diatomic pair potentials to generate the potential energy surfaces arising from vibronic coupling of the Jahn-Teller active vibrational modes with the triply degenerate excited ${ }^{1} T_{1 u}$ electronic state. The vibronic coupling in the solid state is developed from the behavior of the gas phase $\mathrm{Zn} \cdot \mathrm{RG}_{n}$ van der Waals cluster species and outlined in

TABLE I. Photophysical characteristics of the luminescence observed in the Zn/RG $\urcorner$ matrix $\urcorner$ systems $\neg$ at $\neg$ 9 $\urcorner$ K. $\urcorner$ For $\urcorner$ comparison $\urcorner$ the $\urcorner$ singlet $\left(4 s 4 p{ }^{1} P_{1} \rightarrow 4 s^{2}{ }^{1} S_{0}\right)$ and triplet $\left(4 s 4 p{ }^{3} P_{1} \rightarrow 4 s^{2}{ }^{1} S_{0}\right)$ resonance transitions of atomic zinc in the gas phase are also given as well as assignments of the matrix emission bands. Spin singlet and triplet assignments of the observed emission bands are given as “'Sing”' and “Trip', respectively.

\begin{tabular}{lcccc}
\hline \hline & $\lambda_{\text {abs }}(\mathrm{nm})$ & $\lambda_{\text {em }}(\mathrm{nm})$ & $\tau(\mathrm{ns})$ & Trans. \\
\hline Zn gas phase $\neg$ & 213.86 & $213.86\urcorner$ & $1.41\urcorner$ & Sing \\
& & $307.59\urcorner$ & $30.4 \mu \mathrm{s}$ & Trip \\
$\mathrm{Zn} / \mathrm{Ar}\urcorner$ & $206.7 \neg$ & $218.7\urcorner$ & $0.93\urcorner$ & Sing \\
& & $238.0\urcorner$ & $1.42\urcorner$ & Sing \\
$\mathrm{Zn} / \mathrm{Kr}\urcorner$ & $239.5\urcorner$ & $1.26\urcorner$ & Sing \\
& $212.4\urcorner$ & $258.7\urcorner$ & $1.63\urcorner$ & Sing \\
& & $315.6\urcorner$ & $>10 \mu \mathrm{s}^{\mathrm{a}}$ & Trip \\
$\mathrm{Zn} / \mathrm{Xe}\urcorner$ & $356.0\urcorner$ & $>10 \mu \mathrm{s}^{\mathrm{a}}$ & Trip \\
& $219.9\urcorner$ & $399.0\urcorner$ & $>10 \mu \mathrm{s}^{\mathrm{a}}$ & Trip \\
\hline \hline
\end{tabular}

${ }^{\mathrm{a}}$ Lower limit estimates of the decay time made using the time correlated single photon counting technique with an excitation repetition rate of 1.042 $\mathrm{MHz}$.

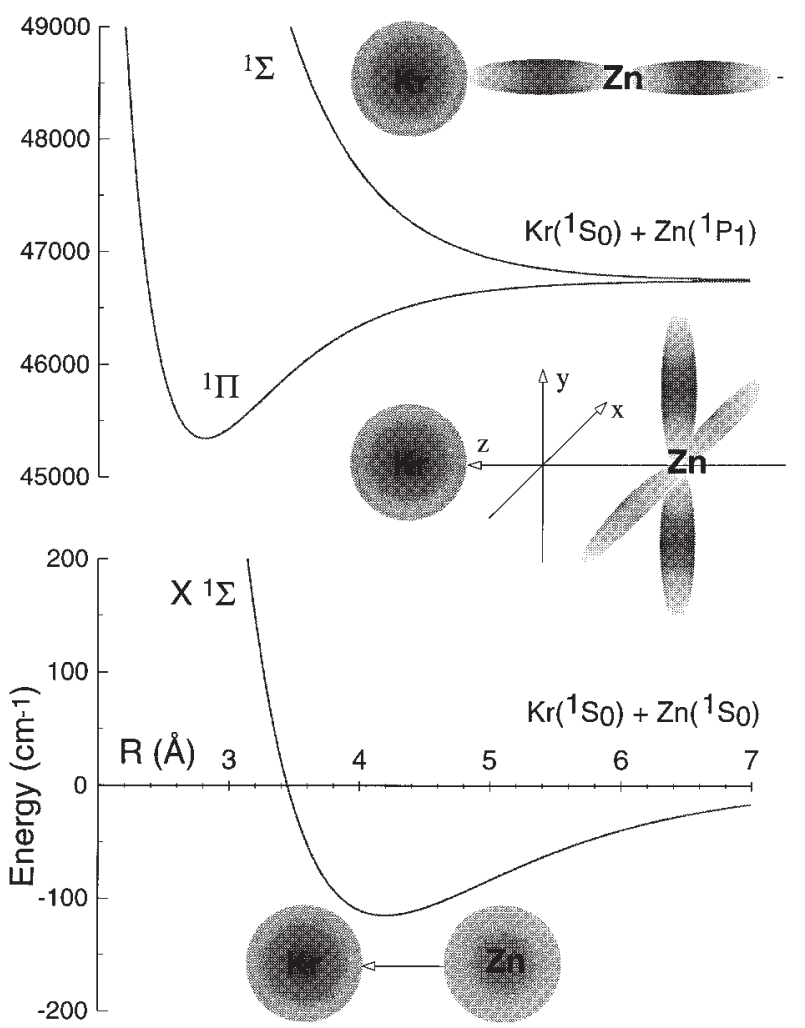

FIG. 2. Potential energy curves for the molecular states of the diatomic van der Waals molecule $\mathrm{Zn} \cdot \mathrm{Kr}$ arising from the interaction of ground $\mathrm{Zn}\left(4 s^{2}{ }^{1} S_{0}\right)$ and excited state $\mathrm{Zn}\left(4 s 4 p^{1} P_{1}\right)$ with krypton. The three plots were generated with the data given in Table II for $\mathrm{Zn} \cdot \mathrm{Kr}$. Note the different energy scales used in the two plots - the energy range in the upper plot is ten times that in the lower.

detail for the $\mathrm{Zn} \cdot \mathrm{Kr}_{n}$ system. Analysis of the theoretical model involves comparison with the luminescence observed in the $\mathrm{Zn} / \mathrm{RG}$ solids.

\section{METHOD}

The central idea on which the theoretical method relies is that the interaction between the optically active guest and the host lattice is limited to those host atoms in the direct vicinity of the guest, i.e., a localized model. The overall energy of the system is then given by the pairwise sum of the individual guest-host interactions considered, for which diatomic potentials are used. The diatomic $(\mathrm{Zn} \cdot \mathrm{RG})$ interaction potentials used in the simulation of the optical spectroscopy of atomic $\mathrm{Zn}$ isolated in rare gas clusters $\left(\mathrm{Zn} \cdot \mathrm{RG}_{n}\right)$ and matrices (Zn/RG) were obtained (1) from high level $a b$ initio calculations $^{7}$ for purely repulsive states and (2) extracted from experimental data derived from laser spectroscopy ${ }^{8}$ of the bound molecular states in supersonic expansions. The potential energy curves of the ground $X^{1} \Sigma_{0}$ and excited ${ }^{1} \Pi_{1}$ and ${ }^{1} \Sigma$ states of diatomic $\mathrm{Zn} \cdot \mathrm{Kr}$ are shown in Fig. 2, as well as pictorial representations of the atomic orbital interactions giving rise to the three molecular states.

The key assumptions in the theoretical model employed are (a) all interactions are obtained by the sum of diatomic pair potentials and (b) the atomic electronic angular momen- 
tum $J_{e}$ at the molecular asymptote is conserved in the cluster. The latter assumption can be defended when it is remembered that the electronic structure of atomic zinc is sparse in the ground and excited states ${ }^{9}$ and none of the rare gas atoms have electronic levels in the vicinity of excited atomic $\mathrm{Zn}\left(4 s 4 p{ }^{1} P_{1}\right)$ state. The validity of the first assumptionthe use of gas phase diatomic potentials to predict the spectroscopy in a solid-will be examined in the present work. Previous work on the triatomic $\operatorname{Hg}\left({ }^{3} P_{1}\right) \cdot \operatorname{Ar}_{2}$ complex has already shown that the theoretical method ${ }^{10,11}$ succeeds in simulating the vibronic structure in the resonance twophotonionization (R2PI) spectrum ${ }^{12}$ of Martrenchard-Barra et al. for this cluster species. Initial attempts have successfully been made by Crepin and Tramer to apply this method to the $\mathrm{Hg} / \mathrm{RG}$ matrix systems ${ }^{13}$ signaling the worthwhileness of the present endeavor. R2PI spectroscopy of a variety of $\mathrm{M} \cdot \mathrm{RG}_{n}$ complexes, currently being undertaken by Breckenridge and co-workers, ${ }^{14}$ will allow a check to be made of the validity of the method in metal atom rare gas cluster systems other than mercury.

\section{A. Ground $X$ electronic state}

The ground $X$ electronic state of a $\mathrm{Zn} \cdot \mathrm{RG}_{n}$ cluster is simple to evaluate due to the spherical symmetry of the atomic electronic angular momentum (EAM) $J_{e}=0$ at the asymptotic limit, i.e., $\mathrm{Zn}\left(4 s^{2}{ }^{1} S_{0}\right)$. The cluster interaction potential $W_{X}(R)$ is given directly by the following sum of the diatomic $\mathrm{Zn} \cdot \mathrm{RG}$ and RG-RG ground electronic state potentials

$$
W_{X}(R)=\sum_{k=1}^{n} V_{\mathrm{Zn} \cdot \mathrm{RG}}^{X}\left(R_{k}\right)+\sum_{i=1}^{m} V_{\mathrm{RG}-\mathrm{RG}}^{X}\left(r_{i}\right), \neg
$$

where $n$ is the number and $R_{k}$ the length of zinc atom-rare gas atom bonds in the cluster. The second term in Eq. (1) includes the energy of the $m \mathrm{RG}-\mathrm{RG}$ bonds in the $\mathrm{Zn} \cdot \mathrm{RG}_{n}$ cluster providing the correct total energy of the system. However, in optical transitions of the cluster which do not involve a variation of the rare gas distances $r_{i}$, this term contributes equally to the two levels coupled in the transition and hence cancels out in calculation of the transition energy. In the expressions which follow, this RG-RG term will not be written explicitly but in any situation where a variation of the RG-RG distance from equilibrium occurs, it will be included in the calculation of the total energy.

\section{B. First excited electronic state}

The energy of the excited $\mathrm{Zn}\left(4 s 4 p{ }^{1} P_{1}\right) \cdot \mathrm{RG}_{n}$ cluster states are considerably more difficult to obtain than the ground state due to the axial symmetry of $J_{e}=1$, the electronic angular momentum of $\operatorname{Zn}\left({ }^{1} P_{1}\right)$ at the molecular asymptote. The method used is that developed by Beswick and co-workers ${ }^{15}$ and their notation is followed throughout this paper. In the excited states the EAM of the cluster is obtained by projection of the diatomic Zn-RG axis (defined as $Z_{k}$ ) onto the cluster axis (defined as $Z$ ) a task accomplished with the Wigner rotation ${ }^{16}$ matrices $D(\phi, \theta, 0)$ over the Euler angles $\phi$ and $\theta$. In Hund's case (a) nomenclature, the molecular states ${ }^{17}$ of the diatomic $\mathrm{Zn} \cdot \mathrm{RG}$ complexes, arising from the approach of $\mathrm{Zn}\left({ }^{1} P_{1}\right)$ to a closed-shell rare gas atom, have, as illustrated in Fig. 2, pure $\Pi$ and $\Sigma$ designations arising from the projection of the atomic angular momentum of $J=1$ on the internuclear axis corresponding to $p_{x}, p_{y}$ and $p_{z}$ orbital occupancy respectively on atomic $\mathrm{Zn}$. The cluster potentials are generated by applying the Wigner rotation matrices to the diatomic potentials using Hund's case (a) quantum numbers as the diatomic basis set.

In the general solution ${ }^{18}$ for $J=1$, the three atomic $p$-orbitals give rise to the following cluster states:

$$
\begin{aligned}
\left(p_{z}\right) W_{1}(R)= & \sum_{k=1}^{n} \cos ^{2} \theta_{k} V_{\Sigma}\left(R_{k}\right)+\sin ^{2} \theta_{k} V_{\Pi}\left(R_{k}\right), \\
\left(p_{x}\right) W_{2}(R)= & \sum_{k=1}^{n} \sin ^{2} \theta_{k} \cos ^{2} \phi_{k} V_{\Sigma}\left(R_{k}\right) \\
& +\left[\cos ^{2} \theta_{k} \cos ^{2} \phi_{k}+\sin ^{2} \phi_{k}\right] V_{\Pi}\left(R_{k}\right), \\
\left(p_{y}\right) W_{3}(R)= & \sum_{k=1}^{n} \sin ^{2} \theta_{k} \sin ^{2} \phi_{k} V_{\Sigma}\left(R_{k}\right) \\
& +\left[\cos ^{2} \theta_{k} \sin ^{2} \phi_{k}+\cos ^{2} \phi_{k}\right] V_{\Pi}\left(R_{k}\right),
\end{aligned}
$$

where $n$ is number of zinc-rare gas bonds in the cluster with $V_{\Pi}(R)$ and $V_{\Sigma}(R)$ the ${ }^{1} \Pi_{1}$ and ${ }^{1} \Sigma$ state potentials of the diatomic $\mathrm{Zn} \cdot \mathrm{RG}$ complexes. The meaning of the other variables present in Eqs. (2)-(4) are depicted in the upper portion of Fig. 3. In the cluster based coordinate system shown in Fig. 3, having the metal atom at its origin, $\theta_{1}$ is the angle subtended between the cluster $Z$ axis and the rare gas atom labeled 1 , and $\phi_{1}$ is the angle existing on the $x$ axis by projecting the $\mathrm{Zn}-\mathrm{RG}_{1}$ axis onto the cluster $X-Y$ plane. $R_{1}$ is the distance of the zinc atom to rare gas atom 1 while $R_{\mathrm{CM}}$ is the distance of the zinc atom to the center-of-mass of the rare gas cluster.

Expressions for the triatomic $\mathrm{Hg} \cdot \mathrm{Ar}_{2}$ complex are given in the work by Beswick and co-workers, ${ }^{10}$ as well as for the tetrameric $\left(\mathrm{Zn} \cdot \mathrm{RG}_{3}\right)$ zinc rare gas clusters by Kaup and Breckenridge. ${ }^{2}$ The specific forms of the excited state energies of the $\mathrm{Zn}\left({ }^{1} P_{1}\right) \cdot \mathrm{RG}_{4}$ and $\mathrm{Zn}\left({ }^{1} P_{1}\right) \cdot \mathrm{RG}_{5}$ clusters will, however, be developed and presented with the objective of obtaining the behavior of the ${ }^{1} P_{1}$ state of atomic $\mathrm{Zn}$ in the solid rare gases, denoted hereafter as $\operatorname{Zn}\left({ }^{1} T_{1 u}\right) / \mathrm{RG}$.

\section{RESULTS}

\section{A. $\mathbf{M} \cdot \mathrm{RG}_{4}$}

In the highest symmetry approach ${ }^{19}$ for the pentatomic cluster $\left(C_{4 v}\right)$, motion of the $\mathrm{Zn}$ atom is restricted to a single axis, defined as the $Z$ axis, perpendicular to the plane of the rare gas atoms shown on the bottom of Fig. 3. For this complex, $n=4$ and under the high symmetry approach chosen a single variable $R$ replaces a maximum of four $R_{k}$ values while $\theta$ suffices for the values of $\theta_{k}$. Using the angles $\phi_{k}$ 

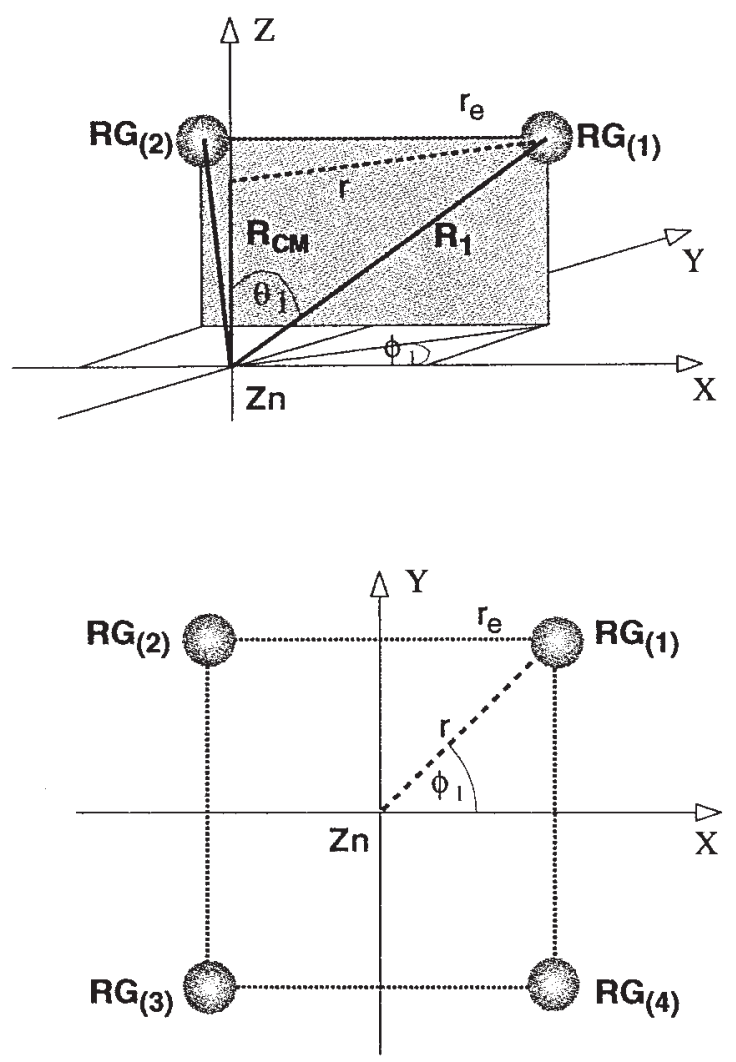

FIG. 3. Diagrams of the metal atom-based coordinate system illustrating the meaning of the variables which are used in Eqs. (2)-(4) for the calculation of the excited state energies of the $\mathrm{Zn}\left(4 s 4 p^{1} P_{1}\right) \cdot \mathrm{RG}_{4}$ cluster species. In the frontal view shown in the upper diagram, the pair of rare gas atoms at the front of the cluster are not displayed to allow identification of the Euler angles $\theta$ and $\phi$. The lower diagram is a "top-view" taken along the cluster $\mathrm{Z}$ axis, i.e., the axis of approach of the $\mathrm{Zn}$ atom to the square of rare gas atoms. $r_{c}$ is rare gas dimer bond length while $r$ is the distance of each RG atom to the center-of-mass of the cluster

$=\pi / 4,3 \pi / 4,5 \pi / 4$, and $7 \pi / 4$ shown in Fig. 3, evaluation of the sums appearing in the general equations (2)-(4) yield the following expressions:

$$
\begin{aligned}
& \left.W_{A 1}(R)=4\left[\cos ^{2} \theta V_{\Sigma}(R)+\sin ^{2} \theta V_{\Pi}(R)\right],\right\urcorner \\
& \left.W_{E}(R)=2\left[\sin ^{2} \theta V_{\Sigma}(R)+\left[\cos ^{2} \theta+1\right] V_{\Pi}(R)\right],\right\urcorner
\end{aligned}
$$

for the ${ }^{1} A_{1}\left(p_{z}\right)$ and the doubly degenerate ${ }^{1} E\left(p_{x}, p_{y}\right)$ electronic states arising from the perpendicular approach of $\mathrm{Zn}\left({ }^{1} P_{1}\right)$ to a square planar arrangement of a cluster of four rare gas atoms.

Plots of the ${ }^{1} A_{1}$ and ${ }^{1} E$ states of $\mathrm{Zn} \cdot \mathrm{Kr}_{4}$ are shown in Fig. 4, calculated from Eqs. (5) and (6) as a function of distance $R_{\mathrm{CM}}$ of the $\mathrm{Zn}$ atom to the center-of-mass of the square of $\mathrm{Kr}$ atoms. Calculations are performed with neighboring $\mathrm{Kr}$ atom distances set at the equilibrium internuclear separation $\left(r_{e}\right)$ of the ground electronic state of $\mathrm{Kr}$ dimer. With the Kr-Kr distances set at $r_{e}$, the calculations shown in Fig. 4 are performed linear in the $\mathrm{Zn}$ to Kr cluster center-ofmass distance $R_{\mathrm{CM}}$. The values of $R$ and $\theta$ appearing in Eqs. (5) and (6) are obtained from the trigonometric relations $R$ $=\sqrt{R_{\mathrm{CM}}^{2}+r^{2}}$ and $\theta=\sin ^{-1}(r / R)$ in which $r=r_{e} / \sqrt{2}$ is the

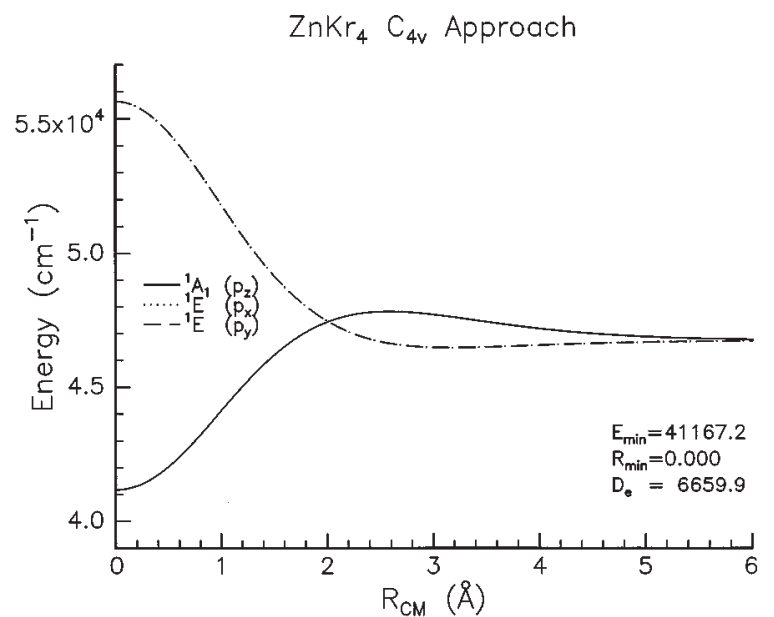

FIG. 4. Potential energy curves of the ${ }^{1} A_{1}$ state and doubly degenerate ${ }^{1} E$ electronic states calculated from Eqs. (5) and (6) as a function of the centerof-mass distance $R_{\mathrm{CM}}$ for the perpendicular approach of atomic $\mathrm{Zn}$ in $p_{z}$ and $p_{x}, p_{y}$ orbitals, respectively, to a planar cluster of $\mathrm{Kr}$ atoms. The four $\mathrm{Kr}$ atoms in the cluster are arranged in a square planar configuration and separated by $4.017 \AA$, the equilibrium internuclear separation of the ground electronic state of $\mathrm{Kr}_{2}$.

distance of each $\mathrm{Kr}$ atom to the center-of-mass of the $\mathrm{Kr}_{4}$ cluster. In turn, the diatomic potentials $V_{\Pi}(R)$ and $V_{\Sigma}(R)$ appearing in Eqs. (5) and (6) are calculated as a function of the $\mathrm{Zn}-\mathrm{Kr}$ internuclear distance $R$ using the Morse and repulsive exponential functions, respectively, listed in Table II for the ${ }^{1} \Pi_{1}$ and ${ }^{1} \Sigma$ state potentials of $\mathrm{Zn} \cdot \mathrm{Kr}$.

It is evident in Fig. 4 that the $C_{4 v}$ approach of $\mathrm{Zn}\left(p_{z}\right)$ in the ${ }^{1} A_{1}$ state is slightly repulsive up to $2.5 \AA$ away from the

TABLE II. Morse $D_{e}\left[1-e^{-\beta\left(R-R_{e}\right)}\right]^{2}$ and repulsive exponential ${ }^{\mathrm{a}} A e^{-\beta(R)}$ functions used as bound and free state pair potentials respectively for rare gas-rare gas interactions and atomic zinc-rare gas atom interactions. Data

\begin{tabular}{|c|c|c|c|c|}
\hline Diatomic $\neg$ & $\left.D_{e}\left(\mathrm{~cm}^{-1}\right)\right\urcorner$ & $R_{e}(\AA)$ & $\left.\beta\left(\AA^{-1}\right)\right\urcorner$ & $A\left(\times 10^{-6} \mathrm{~cm}^{-1}\right)$ \\
\hline $\operatorname{Ar}-\operatorname{Ar}\left(X^{1} \Sigma\right) \neg$ & 99.2 & 3.761า & ר1.685 & $\ldots$ \\
\hline $\left.\mathrm{Kr}-\mathrm{Kr}\left(X^{1} \Sigma\right)\right\urcorner$ & $138.4 \neg$ & 4.017 & ᄀ1.604 & $\ldots$ \\
\hline $\left.\operatorname{Xe}-X e\left(X^{1} \Sigma\right)\right\urcorner$ & 196.24 & 4.3634ᄀ & ר1.509 & $\ldots$ \\
\hline $\left.\operatorname{ZnAr}\left(X^{1} \Sigma\right)\right\urcorner$ & ר5 & ר.18 & $1.001^{\mathrm{a}}$ & $\ldots$ \\
\hline $\left.\operatorname{ZnKr}\left(X^{1} \Sigma\right)\right\urcorner$ & 115 & $4.2\urcorner$ & ר.922 & $\ldots$ \\
\hline $\left.\operatorname{ZnXe}\left(X^{1} \Sigma\right)\right\urcorner$ & 162 & $4.4 \neg$ & ר.828 & $\ldots$ \\
\hline $\left.\operatorname{Zn} \operatorname{Ar}\left({ }^{1} \Pi\right)\right\urcorner$ & ᄀ700 & $2.97 \neg$ & ר1.429 & $\ldots$ \\
\hline $\left.\operatorname{ZnKr}\left({ }^{1} \Pi\right)\right\urcorner$ & ᄀ1400 & $2.80\urcorner$ & $1.594 \neg$ & $\ldots$ \\
\hline $\left.\operatorname{ZnXe}\left({ }^{1} \Pi\right)\right\urcorner$ & $3241\urcorner$ & 2.83ᄀ & ר1.649 & $\ldots$ \\
\hline $\left.\operatorname{ZnAr}\left({ }^{3} \Pi\right)\right\urcorner$ & $487 \neg$ & $3.23\urcorner$ & 1.378 ᄀ & $\ldots$ \\
\hline $\left.\operatorname{ZnKr}\left({ }^{3} \Pi\right)\right\urcorner$ & רי800 & $3.1\urcorner$ & $1.692\urcorner$ & $\ldots$ \\
\hline $\left.\operatorname{ZnXe}\left({ }^{3} \Pi\right)\right\urcorner$ & ᄀ1400 & $3.0 \neg$ & 1.75 & $\ldots$ \\
\hline $\operatorname{ZnAr}\left({ }^{1} \Sigma\right)^{\mathrm{a}}$ & $\ldots$ & $\ldots$ & $1.615 \neg$ & 0.50 \\
\hline $\operatorname{ZnKr}\left({ }^{1} \Sigma\right)^{\mathrm{a}}$ & $\ldots$ & $\ldots$ & ר1.600 & 0.55 \\
\hline $\operatorname{ZnXe}\left({ }^{1} \Sigma\right)^{\mathrm{a}}$ & $\cdots$ & $\ldots$ & $1.725\urcorner$ & 1.00 \\
\hline $\operatorname{ZnAr}\left({ }^{3} \Sigma\right)^{a}$ & $\cdots$ & $\ldots$ & 1.588ᄀ & 0.54 \\
\hline $\operatorname{ZnKr}\left({ }^{3} \Sigma\right)^{\mathrm{a}}$ & $\ldots$ & $\ldots$ & ר1.558 & 0.598 \\
\hline $\mathrm{ZnXe}\left({ }^{3} \Sigma\right)^{\mathrm{a}}$ & $\ldots$ & $\ldots$ & ᄀ1.700 & 1.079 \\
\hline
\end{tabular}
are that quoted in Ref. 2.

${ }^{\text {a The }} \beta$ coefficient of ground state $\mathrm{Zn} \cdot \mathrm{Ar}$ is obtained by extrapolating the corresponding $\mathrm{Zn} \cdot \mathrm{Kr}$ and $\mathrm{Zn} \cdot \mathrm{Xe}$ values based on the trends exhibited in the $X^{1} \Sigma$ states of the RG-RG systems for which more accurate data are available. 
center-of-mass of the planar $\mathrm{Kr}_{4}$ species. Thereafter it is strongly attractive and exhibits a dissociation energy in the “'inserted”' $D_{4 h}$ square planar structure (i.e., at $R=0$ ) of $6659.9 \mathrm{~cm}^{-1}$. Relative to the molecular asymptote at $46745.4 \mathrm{~cm}^{-1}$ the binding energy of the ${ }^{1} A_{1}$ state is $5578.2 \mathrm{~cm}^{-1}$ which is only $22 \mathrm{~cm}^{-1}$ less than four times the binding energy of the ${ }^{1} \Pi_{1}$ state of diatomic $\mathrm{Zn} \cdot \mathrm{Kr}$ $\left(1400 \mathrm{~cm}^{-1}\right)$. This similarity in the binding energies arises because the distance $r$ of each $\mathrm{Kr}$ atom from the center-ofmass of the square $\mathrm{Kr}_{4}$ cluster (having the $\mathrm{Kr}$ atoms separated by the bond length of the $\mathrm{Kr}$ dimer) is $2.84 \AA$, which, as quoted in Table II, matches almost exactly the equilibrium internuclear separation $r_{e}=2.80 \AA$ of the excited ${ }^{1} \Pi_{1}$ state of diatomic $\mathrm{Zn} \cdot \mathrm{Kr}$. It will be shown in what follows that this inserted $\mathrm{Zn} \cdot \mathrm{RG}_{4}$ cluster having a $D_{4 h}$ square planar structure is the species which allows the greatest stabilization in the excited $\mathrm{Zn}\left({ }^{1} P_{1}\right) \cdot \mathrm{RG}_{n}$ states, when the rare gas distances are those of their ground state dimers. In contrast to the ${ }^{1} A_{1}$ state, the ${ }^{1} E$ states are initially slightly attractive but as $\mathrm{Zn}\left(p_{x}, p_{y}\right)$ approaches the RG cluster closer than $3 \AA$ the interaction is repulsive reaching a maximum at the inserted $D_{4 h}$ square planar geometry.

Expressions corresponding to Eqs. (5) and (6) can be obtained from Eqs. (2)-(4) for the perpendicular $C_{n v}$ approach of $\mathrm{Zn}\left({ }^{1} P_{1}\right)$ to a planar cluster composed of any number $n$ of rare gas atoms. The equations for the ${ }^{1} A_{1}\left(p_{z}\right)$ and ${ }^{1} E\left(p_{x}, p_{y}\right)$ states in the $\mathrm{Zn} \cdot \mathrm{Kr}_{3}, \mathrm{Zn} \cdot \mathrm{Kr}_{5}$, and $\mathrm{Zn} \cdot \mathrm{Kr}_{6}$ cluster species are given in Appendix A. The expressions for the ${ }^{1} A_{1}\left(p_{z}\right)$ states are equivalent to Eq. (5) with the multiplicative factors 3,5 , and 6 , respectively, replacing the value of 4 which appears in Eq. (5). In pursuing the luminescence characteristics of the $C_{n v} \mathrm{Zn} \cdot \mathrm{RG}_{n}$ cluster species only the ${ }^{1} A_{1}$ state will be considered as it will have a lower energy than the repulsive ${ }^{1} E$ state. Figure 5 indicates that the $\mathrm{Zn} \cdot \mathrm{Kr}_{n}$ cluster exhibiting the largest stabilization energy is the $\mathrm{Zn} \cdot \mathrm{Kr}_{4}$ species, shown by the solid trace. As in Fig. 4, the $\mathrm{Kr}$ atoms in the clusters are separated by the equilibrium internuclear distance of the ground electronic state of $\mathrm{Kr}$ dimer.

\section{B. $M \cdot \mathbf{R G}_{5}$}

Only a minor extension of Eqs. (5) and (6) pertaining to $\mathrm{M} \cdot \mathrm{RG}_{4}$ must be made to obtain the energies of the $\mathrm{Zn} \cdot \mathrm{RG}_{5}$ complex in $C_{4 v}$ symmetry, i.e., the insertion of the zinc atom into the base of a square pyramid structure composed of five rare gas atoms. This is accomplished with the addition to the $\mathrm{Zn} \cdot \mathrm{Kr}_{4}$ expressions of a $V_{\Sigma}(R+r)$ term for the $A$ electronic state [Eq. (5)] and a $V_{\Pi}(R+r)$ term for the $E$ state [Eq. (6)], i.e.,

$$
\begin{aligned}
W_{A 1}(R)= & 4\left[\cos ^{2} \theta V_{\Sigma}(R)+\sin ^{2} \theta V_{\Pi}(R)\right]+V_{\Sigma}(R+r), \\
W_{E}(R)= & 2\left[\sin ^{2} \theta V_{\Sigma}(R)+\left[\cos ^{2} \theta+1\right] V_{\Pi}(R)\right] \\
& \left.+V_{\Pi}(R+r),\right\urcorner
\end{aligned}
$$

where $r$ is the distance from the nucleus of the apical rare gas atom to the base of the square pyramid of the rare gas five structure. $R$ is now the distance from $\mathrm{Zn}$ to the center of

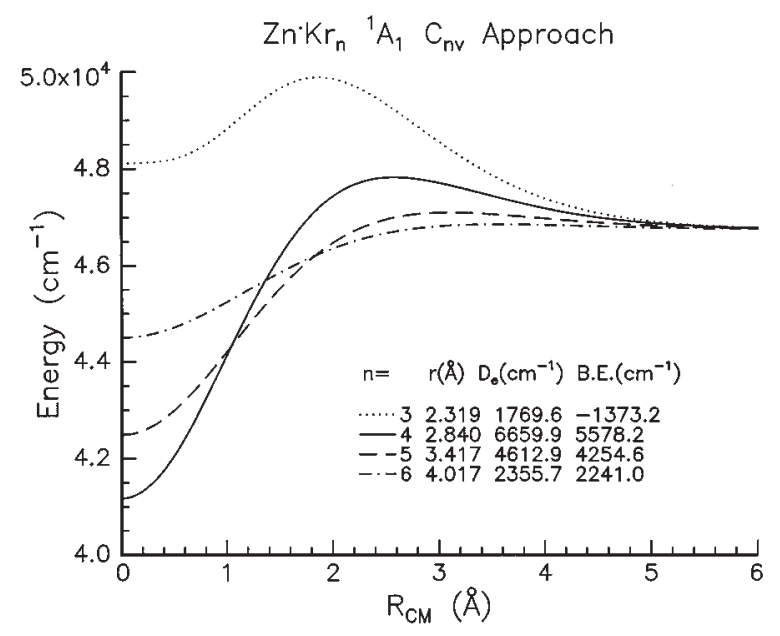

FIG. 5. Potential energy curves, calculated for the perpendicular $C_{n v}$ approach of atomic $\mathrm{Zn}\left(p_{z}\right)$ to planar rare gas clusters made up of the specified number $n$ of $\mathrm{Kr}$ atoms, indicating the particular stability of the $\mathrm{Zn} \cdot \mathrm{Kr}_{4}$ species. The $\mathrm{Kr}$ atoms in the clusters are arranged to form regular planar structures and, as in Fig. 4, are separated by the bond length of ground state $\mathrm{Kr}$ dimer. The $r$ values listed are the distances from any of the $\mathrm{Kr}$ atoms to the center-of-mass of the cluster as calculated from the expressions given in Appendix B. For the square planar $\mathrm{Kr}_{4}$ species $r=2.84 \AA$ very similar to 2.8 $\AA$, the equilibrium internuclear separation of the excited ${ }^{1} \Pi$ state of $\mathrm{Zn} \cdot \mathrm{Kr}$, which explains the particular stability of the $\mathrm{Zn} \cdot \mathrm{Kr}_{4}$ species.

the base of the square pyramid of Kr atoms. The value of $r$ which allows for the formation of eight equal RG-RG bonds in $\mathrm{Zn}\left({ }^{1} P_{1}\right) \cdot \mathrm{RG}_{5}$, can be approximated very accurately by simply taking half of the lattice parameter of the unit cell of the pure rare gas solids. The exact value of $r$ for the equilibrium geometries of the rare gas clusters is, from trigonometry, $r_{e} / \sqrt{2}$ where $r_{e}$ is the equilibrium internuclear separation of gas phase rare gas dimers quoted in Table II in their ground electronic states.

Energy plots of the ${ }^{1} A_{1}\left(p_{z}\right)$ and the ${ }^{1} E\left(p_{x}, p_{y}\right)$ electronic states of the square pyramidal $\mathrm{Zn} \cdot \mathrm{Kr}_{5}$ cluster species are shown in Fig. 6. It is evident from a comparison with Fig. 4 that the stabilization energy of the ${ }^{1} A_{1}$ state of the $\mathrm{Zn} \cdot \mathrm{Kr}_{5}$ complex in $C_{4 v}$ symmetry is much less than that of the corresponding state in $\mathrm{Zn} \cdot \mathrm{Kr}_{4}$. Moreover, the energy minimum is no longer at the center of the four $\mathrm{Kr}$ atoms forming the base of the square pyramid but $0.58 \AA$ away from it. The reason for this large difference between the ${ }^{1} A_{1}$ states of the $\mathrm{Zn} \cdot \mathrm{Kr}_{4}$ and $\mathrm{Zn} \cdot \mathrm{Kr}_{5}$ species originates from the repulsive $\Sigma$ interaction which the additional apical $\mathrm{Kr}$ atom in the $\mathrm{Zn} \cdot \mathrm{Kr}_{5}$ species has with the approaching $\mathrm{Zn}\left(p_{z}\right)$ orbital. Because this $\Sigma$ interaction is purely repulsive, the square pyramidal $\mathrm{Zn} \cdot \mathrm{Kr}_{5}$ species would dissociate in the ${ }^{1} A_{1}$ state with the loss of the apical $\mathrm{Kr}$ atom and the breakage of four RG-RG bonds but resulting in the formation of the more stable inserted square planar $\mathrm{Zn} \cdot \mathrm{Kr}_{4}$ cluster. While a ${ }^{1} A_{1}$ state $\mathrm{Zn} \cdot \mathrm{Kr}_{5}$ cluster of this geometry would be unstable in the gas phase, it is of pivotal importance in setting up a solid state $\mathrm{Zn}\left(p_{z}\right) / \mathrm{RG}$ calculation as it represents the dominant interaction energy associated with the motion of a guest zinc atom from a substitutional site into an octahedral interstitial site of a face centered cubic rare gas lattice. 


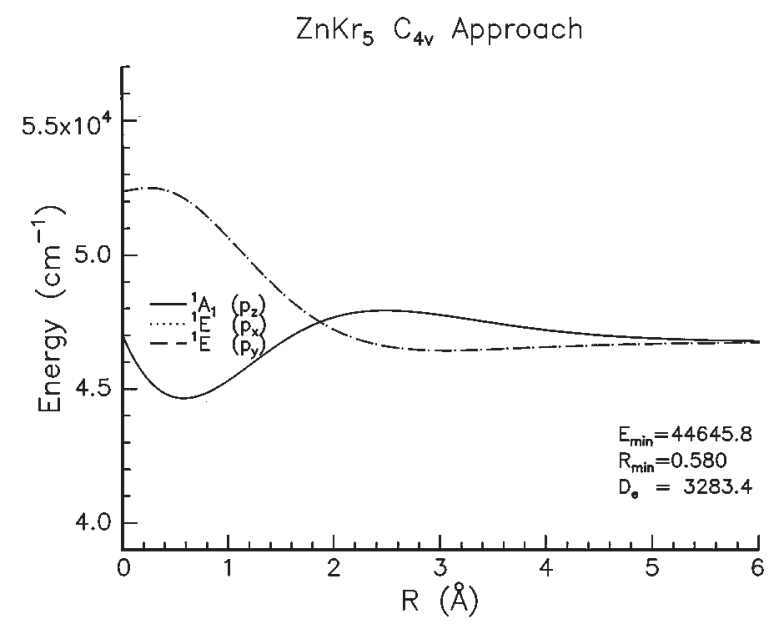

FIG. 6. Potential energy curves for the $C_{4 v} \mathrm{Zn} \cdot \mathrm{Kr}_{5}$ cluster species calculated using Eqs. (7) and (8) as a function of distance $R$ for the ${ }^{1} A_{1}$ and ${ }^{1} E$ states arising from perpendicular approach of atomic $\mathrm{Zn}$ in $\left(p_{z}\right)$ and $\left(p_{x}, p_{y}\right)$ orbitals respectively to the base of a square pyramidal arrangement of five $\mathrm{Kr}$ atoms. The $\mathrm{Kr}$ atoms in the cluster are separated by the equilibrium internuclear separation of ground state $\mathrm{Kr}_{2}$. Note the pronounced destabilization of the ${ }^{1} A_{1}$ state in this cluster compared to the same state of the $\mathrm{Zn} \cdot \mathrm{Kr}_{4}$ species shown in Fig. 4 on the same energy scale.

\section{C. $M / R G$}

Before presenting the details of the solid state calculations, some general comments must be made regarding the method followed. First, because of the van der Waals bond lengths of the Zn-RG dimers and the lattice parameters, $a$, of the solid rare gases, calculations are performed with atomic zinc residing in a substitutional site, i.e., atomic zinc taking the place of a rare gas atom in the solid rare gas lattice. Second, with the rare gas solids having face centered cubic (fcc) packing structures the substitutional sites have cubooctahedral symmetry so that the ${ }^{1} S_{0}$ and ${ }^{1} P_{1}$ atomic states carry the designations ${ }^{1} A_{1 g}$ and ${ }^{1} T_{1 u}$, respectively, in the solid state. Third, under octahedral symmetry equivalent interactions exist for $p_{x}, p_{y}$, and $p_{z}$ orbital occupancy of $\mathrm{Zn}$ and its rare gas host when it resides in a substitutional site. Thus while the zinc atom will have a dominant interaction with its 12 nearest rare gas atom neighbors, the 6 next nearest neighbors must be included to create the required $p$-orbital equivalency. In all the calculations to be presented a minimum of $18 \mathrm{Zn}-\mathrm{RG}$ interactions are considered comprised of 12 nearest neighbors separated in the ground state at the substitutional site diameter $a / \sqrt{2}$ of the solid and 6 next nearest neighbors at a separation equal to the lattice parameter $a$.

In the analysis of the vibronic interactions occurring on the excited ${ }^{1} T_{1 u}$ electronic surface of $\mathrm{Zn}$ in solid rare gases which would be expected to dominate the luminescence, particular attention was given to those modes which allowed the attainment of the $\mathrm{Zn} \cdot \mathrm{RG}_{4}$ species shown in Fig. 5 to produce the largest stabilization energy of the $\mathrm{Zn} \cdot \mathrm{Kr}_{n}$ clusters. An examination of the pair of unit cells of a fcc solid shown in Fig. 7 indicates two possible motions which lead to a dominant interaction of the guest $\operatorname{zinc} \operatorname{Zn}\left(p_{z}\right)$ atom with 4 of its
$\mathrm{Zn}\left({ }^{1} \mathrm{~T}_{1 \mathrm{u}}\right) / \mathrm{Kr}$ lattice distortions

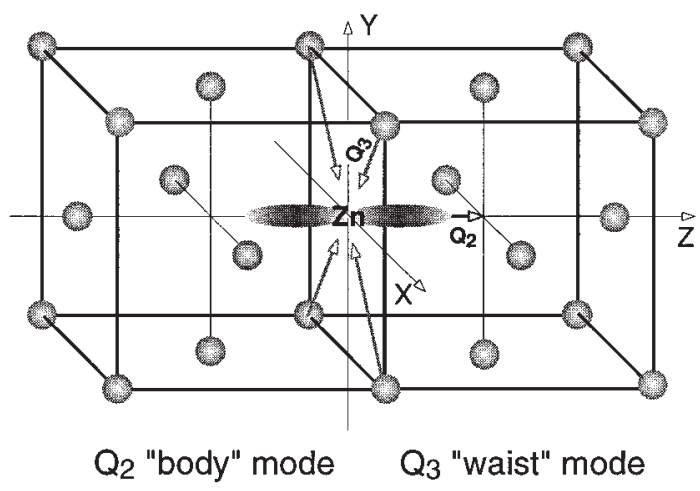

FIG. 7. A pair of unit cells of solid $\mathrm{Kr}$ indicating the possible lattice motions which allow the attainment of the stable $\mathrm{Zn}\left(p_{z}\right) \cdot \mathrm{Kr}_{4}$ species for a $\mathrm{Zn}$ atom originally occupying a substitutional site in the $\mathrm{Kr}$ lattice. $Q_{2}$, called the "'body" mode involves motion of the $\mathrm{Zn}\left(p_{z}\right)$ atom along the $Z$ axis, the body axis of the pair of unit cells shown into an octahedral interstitial site. $Q_{3}$, the "waist" mode, involves an in-phase contraction of the four rare gas atoms at the corners surrounding the $\mathrm{Zn}$ atom on the $X-Y$ plane.

12 nearest neighbor rare gas atoms when isolated in a substitutional site. One motion is simply the contraction of 4 of the nearest neighbor rare gas atoms around the zinc, the other is the motion of the zinc atom into one of the adjacent octahedral interstitial sites. The former motion, described as the "waist" mode, is of tetragonal symmetry and in octahedral $X Y_{6}$ species is labeled $E_{g}$. The other motion in an octahedral complex is the triply degenerate $F_{1 u}$ mode. It will be shown in what follows that these two vibronic modes lead to both a lowering of the energy in the degenerate excited ${ }^{1} T_{1 u}$ electronic state and a reduction of the cubooctahedral symmetry from which the absorption transitions originate, these two properties being the key characteristics ${ }^{20}$ of the Jahn-Teller effect.

\section{Body mode}

Calculations of the stabilization energy arising from the motion indicated as $Q_{2}$ in Fig. 7 and, hereafter referred to as the "body" mode can be obtained from a combination of the $C_{4 v} \mathrm{Zn} \cdot \mathrm{RG}_{4}$ and $\mathrm{Zn} \cdot \mathrm{RG}_{5}$ cluster calculations presented in the preceding sections when it is remembered that the face centered cubic packing in the solid rare gases intrinsically has four-fold symmetry along the $Z$ axis shown in Fig. 7. Details of the $\mathrm{Zn} \cdot \mathrm{RG}$ interactions considered in a calculation of the "body" mode energy are diagrammed in Fig. 8. As indicated in Fig. 8, the motion of the zinc atom along $Q_{2}$, i.e., away from the substitutional site it originally occupies and into an octahedral interstitial site, involves four types of interaction, all of which have four-fold symmetry. Having this symmetry the behavior of the $\operatorname{Zn}\left(p_{z}\right)$ orbital in the $Q_{2}$ vibronic mode of an fcc lattice will be given by Eq. (5) for the ${ }^{1} A_{1}$ state expression

$$
W\left(R_{n}, \theta_{n}\right)=4\left[\cos ^{2} \theta_{n} V_{\Sigma}\left(R_{n}\right)+\sin ^{2} \theta_{n} V_{\Pi}\left(R_{n}\right)\right]
$$

of the $C_{4 v} \mathrm{Zn} \cdot \mathrm{RG}_{4}$ cluster and Eq. (7) for the ${ }^{1} A_{1}$ state of the $C_{4 v} \mathrm{Zn} \cdot \mathrm{RG}_{5}$ cluster. Equation (9) must be evaluated for 


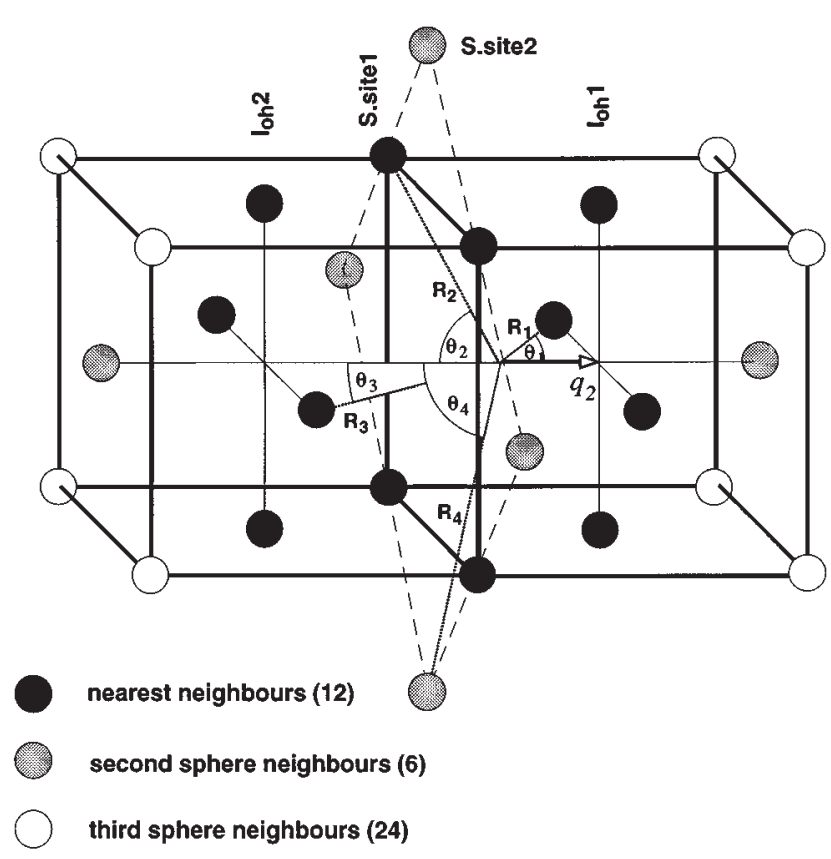

FIG. 8. Details of the distance and angle parameters in the Kr lattice used in the calculation of the "body" mode of $\mathrm{Zn} / \mathrm{Kr}$. The four distinct distance $R$ and angle $\theta$ parameters depicted are required to model the motion of a $\mathrm{Zn}$ atom originally occupying a substitutional site (S.site1) in the Kr lattice into an octahedral interstitial site $I_{\mathrm{oh}} 1$. The different shading used on the atoms in the unit cells represent the rare gas atoms in the first and second spheres surrounding the $\mathrm{Zn}$ atom. The white circles indicate the locations of eight of the rare gas atoms in the third sphere but which are not included in the present calculations.

the specific $R_{n}$ and $\theta_{n}$ variables associated with the four types of interactions indicated in Fig. 8. The identities and functional forms of the four interactions considered in Fig. 8 are as follows;
(1) $I_{\mathrm{oh}} 1 \neg W\left(R_{1}, \theta_{1}\right)+V_{\Sigma}(a-q)$;
(2) S.site1 $\neg W\left(R_{2}, \theta_{2}\right)$;
(3) $\left.I_{\mathrm{oh}} 2\right\urcorner W\left(R_{3}, \theta_{3}\right)+V_{\Sigma}(a+q)$;
(4) S.site $\neg \neg W\left(R_{4}, \theta_{4}\right)$;

where " $a$ " is the lattice parameter of the rare gas unit cell and $q$ is linear displacement of $\operatorname{Zn}\left(p_{z}\right)$ away from the substitutional site. Interaction (1) involves the motion of the $\mathrm{Zn}\left(p_{z}\right)$ atom along the lattice $Z$ axis toward a neighboring octahedral interstitial site $I_{\mathrm{oh}} 1$ composed of five rare gas atoms; interaction (2) is motion of the $\mathrm{Zn}$ away from its four nearest rare gas atoms on the $X Y$ plane S.site1; (3) is the motion away from its other neighboring octahedral interstitial site $I_{\mathrm{oh}} 2$ (five rare gas atoms); and (4) is the motion away from the four next nearest rare gas atoms on the $X Y$ plane S.site2. The calculation is performed linear in $q$, the motion of the zinc atom from the substitutional site, and into an octahedral interstitial site with the four specific $R_{n}$ and $\theta_{n}$ variables calculated for their locations in the unit cells using the trigonometric relationships presented earlier for the $\mathrm{Zn} \cdot \mathrm{RG}_{4}$ cluster. In the solid state $\mathrm{Zn}\left({ }^{1} T_{1 u}\right) / \mathrm{Kr}$ calculations the $\mathrm{Kr}-\mathrm{Kr}$ distances are determined by the lattice parameter of solid $\mathrm{Kr}$ at $4 \mathrm{~K}$ and not the bond length of $\mathrm{Kr}_{2}$ as used

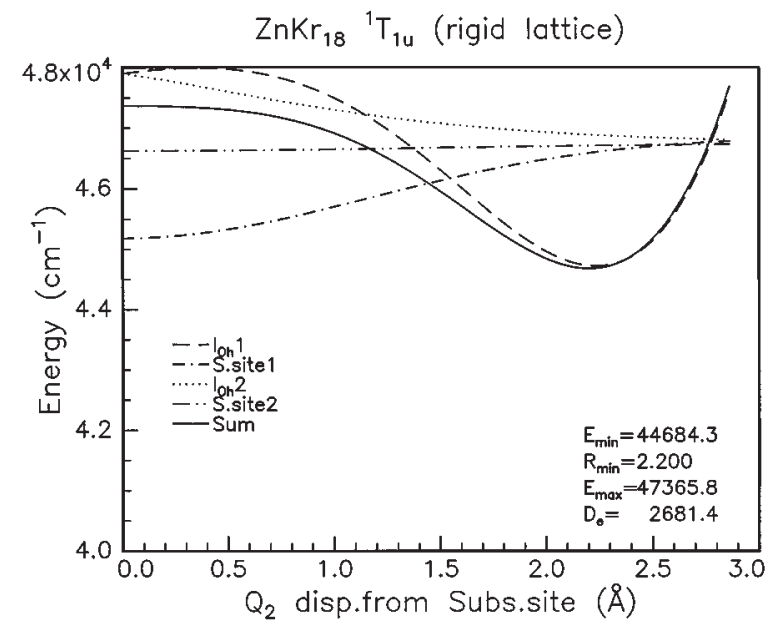

FIG. 9. The excited ${ }^{1} T_{1 u}$ state energy on the body mode $Q_{2}$ of the $\mathrm{Zn} / \mathrm{Kr}$ system obtained from a calculation of the four interactions depicted between the $\mathrm{Zn}$ atom and its neighboring $\mathrm{Kr}$ atoms in Fig. 8. The energies of the specific interactions considered are indicated by the legend and the sum, constituting a resulting interaction of $\mathrm{Zn}$ with 18 nearest $\mathrm{Kr}$ neighbors, is indicated by the solid trace. The energies of the excited singlet ${ }^{1} T_{1 u}$ state is relative to the $4 s 4 p{ }^{1} P_{1}$ level of atomic zinc at $46745.4 \mathrm{~cm}^{-1}$.

earlier in the gas phase clusters; the differences between the two are only minor being 3.988 and $4.017 \AA$, respectively.

The energies associated with the four types of interaction considered in the "body" mode are plotted in Fig. 9 as a function of displacement $Q_{2}$ of the $\operatorname{Zn}\left(p_{z}\right)$ atom away from the substitutional site originally occupied. From the legend in Fig. 9, it is evident that destabilization results from the motion of the zinc atom away from its four nearest rare gas atoms on the $X Y$ plane (S.site1) and to a minor extent from its four next nearest rare gas atoms on this plane (S.site2 in Fig. 9). The two other interactions are stabilized, in particular the repulsive interaction from octahedral interstitial site $I_{\mathrm{oh}} 2$ is reduced and $I_{\mathrm{oh}} 1$ is strongly stabilized. Summing up the $18 \mathrm{Zn}-\mathrm{Kr}$ interactions considered in Eq. (10), the solid curve shown in Fig. 9 indicates that a stabilization energy of $2681.4 \mathrm{~cm}^{-1}$ results from the motion of atomic zinc $\operatorname{Zn}\left(p_{z}\right)$ $2.20 \AA$ away from the substitutional site it originally occupied in the ground ${ }^{1} A_{1 g}$ electronic state of $\mathrm{Zn} / \mathrm{Kr}$.

An examination of the pairs of unit cells shown in Figs. 7 and 8 indicates that the plot of the $Q_{2}$ vibronic mode shown in Fig. 9 is symmetric for motion of the $\mathrm{Zn}$ atom along the lattice $Z$ axis. Thus identical potential energy surfaces result for the motion of the zinc atom from the substitutional site into either of the adjacent octahedral interstitial sites. For this reason only one of them is shown in Fig. 9.

The calculation shown in Fig. 9 is for a rigid Kr lattice, fixed at the lattice parameter of solid krypton. Results of the $\mathrm{Zn} \cdot \mathrm{Kr}_{4}$ and the $\mathrm{Zn} \cdot \mathrm{Kr}_{5}$ cluster calculations shown in Figs. 4 and 6 respectively suggest that a further lowering of energy on the ${ }^{1} T_{1 u}$ electronic state will be achieved as the $\mathrm{Zn}$ atom gets closer to having a complexation number of 4 , i.e., at the center of the octahedral interstitial $\left(I_{\mathrm{oh}}\right)$ site in $\mathrm{Kr}$ at $R$ $=2.82 \AA$. Consideration of the motion of the $\mathrm{Zn}\left(p_{z}\right)$ orbital toward the octahedral interstitial site indicates that this fur- 


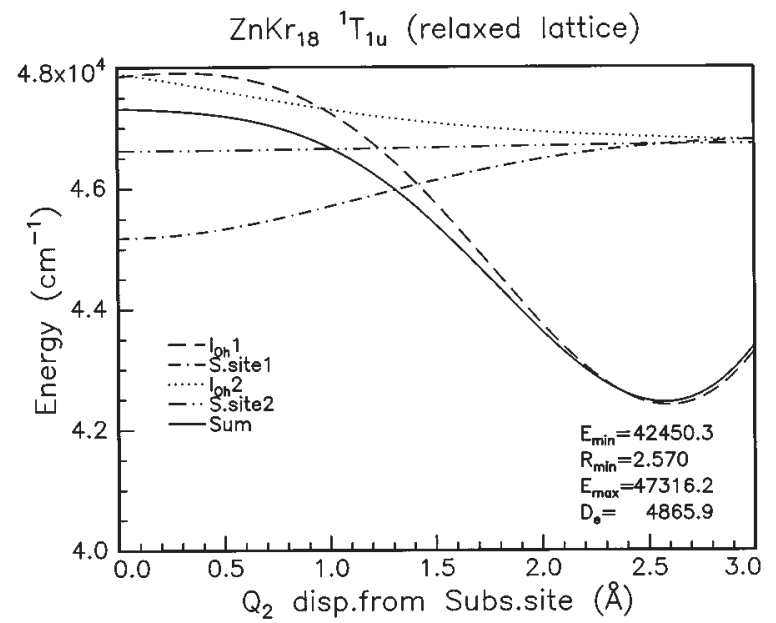

FIG. 10. As Fig. 9 except that the Kr lattice has been relaxed to allow a closer approach of the $\mathrm{Zn}$ atom to the center of the octahedral interstitial site. This is achieved by moving the end $\mathrm{Kr}$ atom, shown on the extreme right in Fig. 8, into the next unit cell. The extent of this motion is ascertained by equalizing the destabilization of the next $\mathrm{Kr}$ unit cell with the increased stabilization arising from having the $\mathrm{Zn}$ atom residing in the octahedral interstitial site.

ther lowering of the energy of the "body" mode is achieved by minimizing the repulsive $\Sigma$ interaction between $\mathrm{Zn}\left(p_{z}\right)$ and the second sphere $\mathrm{Kr}$ atom located on the lattice $Z$ axis. This can be realized by moving the $\mathrm{Kr}$ atom in the center of the end face of the pair of unit cells, shown on the extreme right in Fig. 8, into its adjacent $\mathrm{Kr}$ cell. The minimum of energy on the "body" mode will be achieved when the destabilization induced in the adjacent $\mathrm{Kr}$ unit cell, arising because of forcing the end $\mathrm{Kr}$ atom into it, is equal to the stabilization of the $\Sigma$ state $\mathrm{Zn}\left(p_{z}\right)$ - Kr interaction brought about by this displacement. This equalization is achieved by displacing the $\mathrm{Kr}$ atom $0.8627 \AA$ thereby inducing an $E_{\mathrm{Kr}-\mathrm{Kr}}$ destabilization of $1518 \mathrm{~cm}^{-1}$ in the neighboring $\mathrm{Kr}$ unit cell. However, displacing the $\mathrm{Kr}$ atom by $0.8627 \AA$ produces a stabilization energy of $4865.9 \mathrm{~cm}^{-1}$ on the $\mathrm{Zn} / \mathrm{Kr}$ body mode, with the zinc atom now located, as shown in Fig. 10, at a position $2.57 \AA$ away from the substitutional site originally occupied or only $0.25 \AA$ away from a true $I_{\mathrm{Oh}}$ site of solid $\mathrm{Kr}$.

The destabilization induced in the rare gas lattice by the $Q_{2}$ mode is calculated for the motion of a single rare gas atom from its substitutional site along the body $Z$ axis of a pair of unit cells toward an octahedral interstitial site. The motion can be visualized most easily by replacing the axial $\mathrm{Zn}\left(p_{z}\right)$ orbital shown in Fig. 7 with a spherical RG atom. Seventeen RG-RG interactions are considered, comprised of the 12 nearest RG atoms to the displaced rare gas atom and 5 next nearest neighbors [the absent sixth is now the $\operatorname{Zn}\left(p_{z}\right)$ atom]. The $\mathrm{RG} \cdot \mathrm{RG}_{17}$ calculations are performed by substituting in Eq. (1) the ground state rare gas dimer parameters listed in Table II. The results for the Kr lattice are quoted in the preceding paragraph.

For a comparison with the observed low temperature spectra the emission energy associated with the $Q_{2}$ vibronic
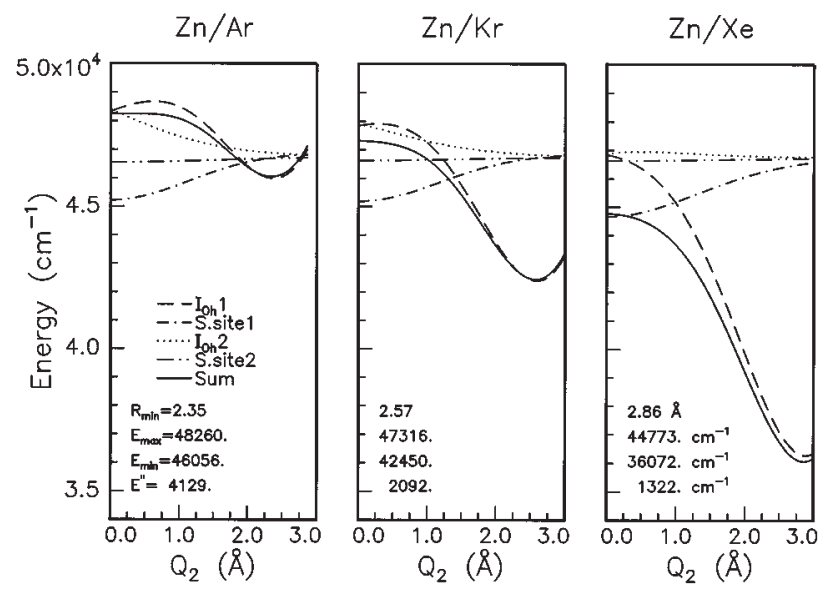

FIG. 11. The excited ${ }^{1} T_{1 u}$ state energies of $\mathrm{Zn} / \mathrm{Ar}, \mathrm{Zn} / \mathrm{Kr}$, and $\mathrm{Zn} / \mathrm{Xe}$ associated with the motion of the $\mathrm{Zn}$ atom along the body of a pair of unit cells of the rare gas solid. The energies of the four interactions considered between the $\mathrm{Zn}$ atom and its surroundings are indicated by the legend. The ground state energy values $\left(E^{\prime \prime}\right)$ at the internuclear separation $R_{\min }$ where the energy minimum $\left(E_{\min }\right)$ of the excited state exists, are required for the calculation of the emission energies and are indicated as $E^{\prime \prime}$ in the figure for each of the $\mathrm{Zn} / \mathrm{RG}$ systems. These values of $E^{\prime \prime}$ shown are calculated from Eq. (1).

mode is obtained from its energy minimum at $R=2.57 \AA$ and assuming a vertical Franck-Condon transition to the ground ${ }^{1} A_{1 g}$ electronic state. Thus the energy of the $\mathrm{Zn}\left({ }^{1} A_{1 g}\right) / \mathrm{Kr}$ ground state must be obtained at the nuclear configurations existing at $R=2.57 \AA$ on the body mode $Q_{2}$. Using the Morse function parameters for the $X$ state of $\mathrm{Zn} \cdot \mathrm{Kr}$ listed in Table II, the ground state energy $E^{\prime \prime}$ at $R$ $=2.57 \AA$ is calculated from Eq. (1) to be $2092 \mathrm{~cm}^{-1}$. The energy of the emission originating from the calculated minimum on the body mode can now be obtained from the following expression:

$$
\left.E_{\mathrm{cm}}=E^{\prime}\left(R_{\min }^{\prime}\right)-E^{\prime \prime}\left(R_{\mathrm{min}}^{\prime}\right)-E_{\mathrm{Kr}-\mathrm{Kr}},\right\urcorner
$$

the last term of which includes the distortion energy in the rare gas lattice. Using the values quoted above for the $\mathrm{Zn} / \mathrm{Kr}$ system, the emission energy is found from Eq. (11) to be $38840 \mathrm{~cm}^{-1}$, a value which compares very well with $38655 \mathrm{~cm}^{-1}$, the center of the $258.7-\mathrm{nm}$ band of $\mathrm{Zn} / \mathrm{Kr}$ listed in Table I.

Following the procedure outlined above for $\mathrm{Zn} / \mathrm{Kr}$, the excited ${ }^{1} T_{1 u}$ state energies for the body mode in relaxed lattices of the $\mathrm{Zn} / \mathrm{Ar}$ and $\mathrm{Zn} / \mathrm{Xe}$ systems were calculated with the pair potential parameters listed in Table II. The results of the calculations on these other systems are shown along with $\mathrm{Zn} / \mathrm{Kr}$ in Fig. 11. The extent of the displacement of the single rare gas atoms in the lattice relaxation occurring on the excited state were 0.9601 and $0.7590 \AA$ in Ar and Xe, respectively, with associated RG lattice destabilization energies of 1457 and $1365 \mathrm{~cm}^{-1}$, respectively, when calculated with the RG-RG data given in Table II. The emission energy resulting from the body mode in the $\mathrm{Zn} / \mathrm{Ar}$ system is calculated to be $40470 \mathrm{~cm}^{-1}$ using the molecular potentials of $\mathrm{Zn} \cdot \mathrm{Ar}$ and Ar-Ar listed in Table II. This value is in only approximate agreement with the 238-nm emission band of $\mathrm{Zn} / \mathrm{Ar}$ ob- 
TABLE III. Energy and distance parameters calculated for the "body" and "waist" vibronic modes used in predicting the emission wavelengths in the matrix Zn/RG systems. Energies $(E)$ are quoted in wavenumber units, distances $(R)$ in Angstroms, and wavelength $(\lambda)$ in nanometers. The predicted emission energies, $E_{\mathrm{em}}$, are calculated from the entries in columns 2, 4, and 5 with Eq. (11). The abbreviation 'n.o.' refers to emission bands which are not observed experimentally in Ar and Xe matrices due to extremely inefficient and extremely efficient intersystem crossing respectively of atomic zinc in these host solids. Energies of the excited singlet and triplet states are obtained relative to the $4 s 4 p{ }^{1} P_{1}$ and $4 s 4 p{ }^{3} P_{1}$ levels of atomic zinc at 46745.4 and 32501.4 $\mathrm{cm}^{-1}$, respectively, in the gas phase.

\begin{tabular}{|c|c|c|c|c|c|c|c|}
\hline Singlet $\neg$ & $E_{\min }^{\prime}$ & $R_{\min }^{\prime}$ & $E^{\prime \prime} @ R_{\min }^{\prime}$ & $E_{\mathrm{RG}-\mathrm{RG}}$ & $E_{\mathrm{em}}$ & $\lambda_{\mathrm{em}}$ & $\lambda_{\mathrm{obs}}$ \\
\hline $\mathrm{Zn} / \mathrm{Ar}(\mathrm{b})$ & ר4056 & ר2.35 & ᄀ4129 & ᄀ1457 & ᄀ470 40 & ᄀ247.10 & 238 \\
\hline (w) & ר 47 063 & ᄀ0.78 & 849 & ר176 & ר־038 46 & $217.21 \neg$ & 218.7 \\
\hline $\mathrm{Zn} / \mathrm{Kr}(\mathrm{b})$ & 42 ᄀ450 & 2.57 & 2092ᄀ & 1518 & ᄀ 38 840 & 257.47ᄀ & 258.7 \\
\hline$(\mathrm{w})$ & ר325 & ר1.19 & 1561 & ר130 & 41 634 & ר240.19 & 239.6 \\
\hline Zn/Xe (b) & ר 36 072 & ר2.86 & 1322ᄀ & ר1365 & ר33 385 & ר299.54 & n.o. \\
\hline (w) & ־906 33 & $1.5 \neg$ & ᄀ2220 & 625 & 31 061 & ר321.95 & n.o. \\
\hline \multicolumn{8}{|l|}{ Triplet } \\
\hline $\mathrm{Zn} / \mathrm{Ar}(\mathrm{b})$ & 34 667ᄀ & ר2.18 & ר3583 & 1623 & 29 461ᄀ & ר39.43 & n.o. \\
\hline (w) & ר700 34 & 0.52 & $-386\urcorner$ & 277 & ר809 34 & ר287.28 & n.o. \\
\hline $\mathrm{Zn} / \mathrm{Kr}$ (b) & 32 ר44 & 2.43 & 1805ר & ר1768 & $28471 \neg$ & ר351.23 & $345^{\mathrm{a}}$ \\
\hline$(\mathrm{w})$ & 32 921 & ᄀ0.89 & $-400 \neg$ & ᄀ197 & 33 124 & ר־301.90 & 315.6 \\
\hline Zn/Xe (b) & 28 ר226 & 2.8ᄀ & ר1213 & $1524\urcorner$ & ר489 25 & ר392.33 & 399 \\
\hline$(\mathrm{w})$ & 28 ר610 & 1.33 & 394ᄀ & 212ᄀ & 28 004ᄀ & ר357.09 & 356 \\
\hline
\end{tabular}

abserved only for temperatures greater than $25 \mathrm{~K}$.

served at $42017 \mathrm{~cm}^{-1}$. The singlet emission is not observed in $\mathrm{Zn} / \mathrm{Xe}$ as total singlet-triplet intersystem crossing occurs in this system, but the calculated emission energy of the "body" mode of the ${ }^{1} T_{1 u}$ state would be $33385 \mathrm{~cm}^{-1}$, i.e., $299.5 \mathrm{~nm}$. The results of the calculations for the "body", mode in the spin singlet $\mathrm{Zn} / \mathrm{RG}$ systems are collected in the upper section of Table III for comparison with the observed emission wavelengths in Ar and $\mathrm{Kr}$.

\section{E. Waist mode}

Calculation of the stabilization energy associated with the "waist" mode in the $\mathrm{Zn}\left({ }^{1} T_{1 u}\right) / \mathrm{RG}$ lattice is considerably simpler than the "body" mode described above, as it involves only a single variable $R$ - the in-phase contraction of four nearest rare gas atoms on a single plane toward the zinc atom. The interactions considered in determining the energy of the "waist"' mode are indicated in Fig. 12 and given by the general expression Eq. (9) for a packing system having four-fold symmetry. Inspection of Fig. 12 indicates the energy of the waist mode is obtained from the following Zn-RG interactions;

(1) The sum of two octahedral interstitial sites $\left(I_{\mathrm{Oh}} 1\right.$ and $I_{\mathrm{Oh}} 2$ ), i.e., two $\mathrm{M} \cdot \mathrm{RG}_{5}$ cluster calculations with the Zn-RG distances $R_{1}$ and $R_{3}$ set at the substitutional site size of the solid rare gas and a value of $\pi / 4$ for the angles $\theta_{1}$ and $\theta_{3}$;

(2) the in-phase contraction of the four rare gas atoms indicated by "S.site1"' in Fig. 12 toward the Zn atom; and

(3) the four next nearest rare gas neighbor atoms on the $X Y$ plane (labeled ' 'S.site2' ' in Fig. 12). Interactions (2) and (3) are given, as for interaction (1), by Eq. (9) but since the angles $\theta_{2}$ and $\theta_{4}$ are now set at $\pi / 2$ in the substitutional site, a simple sum of four diatomic $\mathrm{Zn} \cdot \mathrm{RG}$
${ }^{1} \Pi_{1}$ state potentials suffices in both cases. The former interaction is of variable length $R_{2}$, the latter fixed at, $a$, the lattice parameter of the solid.

From the $R_{e}$ values listed in Table II for the ${ }^{1} \Pi_{1}$ states of the $\mathrm{Zn} \cdot \mathrm{RG}$ species, it is expected that stabilization on this vibronic mode will result from the contraction of the four rare gas atoms (S.site1) from their lattice positions towards the zinc atom. In contrast to the body mode $Q_{2}$, the waist

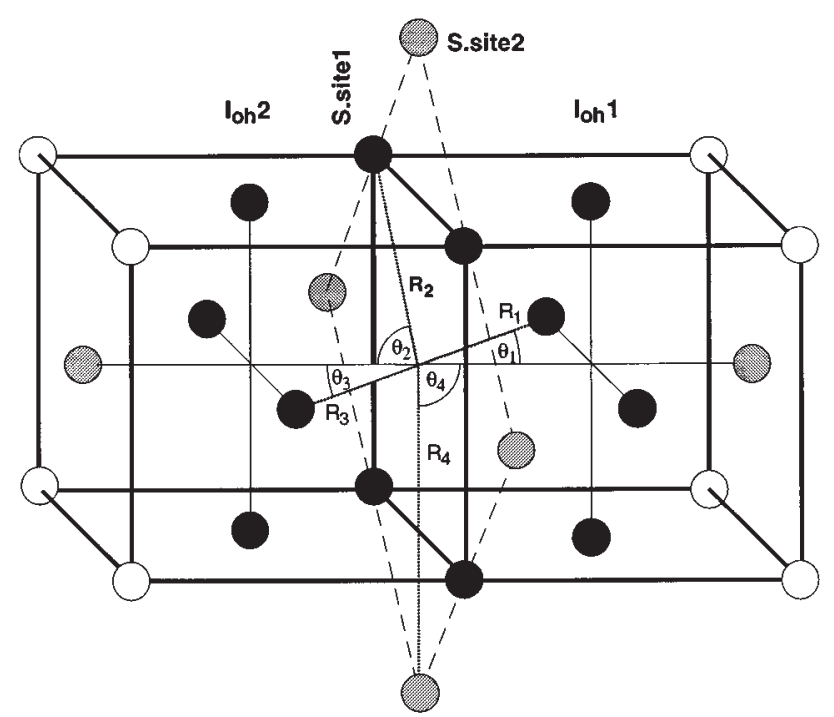

FIG. 12. Details of the distance and angle parameters used in the calculation of the "waist" mode of the $\operatorname{Zn}\left(p_{z}\right) / \mathrm{RG}$ systems. Four types of $\mathrm{Zn}\left(p_{z}\right)$ - RG interactions are considered, three of which are independent of $Q_{3}$ and two having identical distance $R_{1}, R_{3}$, and angle $\theta_{1}, \theta_{3}$ parameters. The fourth interaction, the in-phase contraction on the $X-Y$ plane of the four rare gas atom in on the $\mathrm{Zn}$ atom occupying a substitutional site, has only a single distance parameter $R_{2}$ as a variable. 

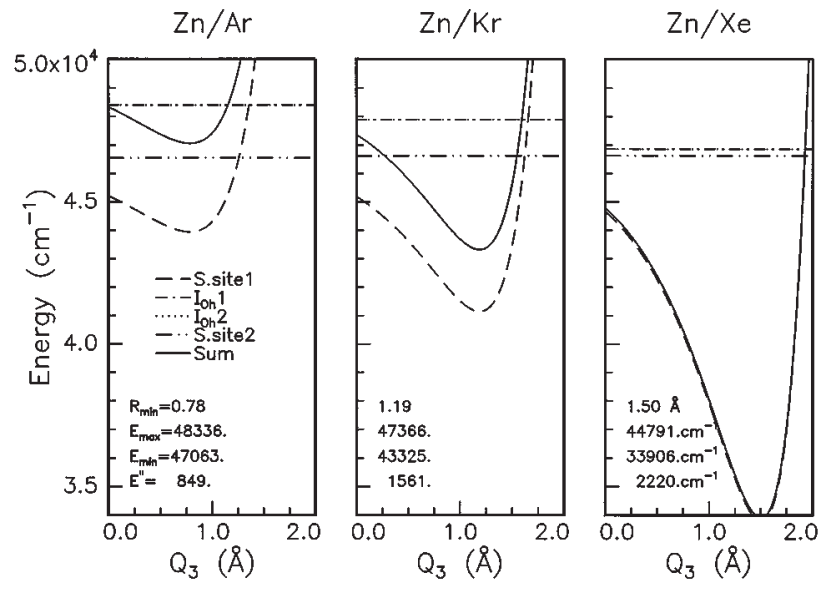

FIG. 13. A comparison of the "waist" mode energies calculated for the four interactions depicted in Fig. 12 for the ${ }^{1} T_{1 u}$ state in $\mathrm{Zn} / \mathrm{Ar}, \mathrm{Zn} / \mathrm{Kr}$, and $\mathrm{Zn} / \mathrm{Xe}$. The meaning of the numerical values in this figure are as in Fig. 11.

mode $Q_{3}$ is not symmetric with respect to the atomic displacements occurring for contraction and expansion along this vibronic mode. Thus contraction will be dominated by the attractive portion of the $\mathrm{Zn} \cdot \mathrm{RG}{ }^{1} \Pi_{1}$ potential while expansion from the substitutional site diameter $Q_{2(R=0)}$ will be repulsive with the gradient, especially at large displacement, dominated by the repulsive wall of the ground state rare gas dimer potentials.

The potential energy curves for this "waist" mode are shown in Fig. 13 for the $\mathrm{Zn} / \mathrm{Ar}, \mathrm{Zn} / \mathrm{Kr}$, and $\mathrm{Zn} / \mathrm{Xe}$ systems and as anticipated, contractions of $0.78,1.19$, and $1.50 \AA$ from the substitutional site sizes of the solid rare gases are obtained for the minima of this mode in $\mathrm{Ar}, \mathrm{Kr}$, and $\mathrm{Xe}$, respectively. Considering that $Q_{3}$ is a cage mode, surprisingly little destabilization of the lattice is induced by the in-phase contraction of the four corner rare gas atoms of the unit cell in their motion on the $X-Y$ plane toward the $\mathrm{Zn}$ atom. This is the case because (a) the $X-Y$ plane is not a close packed plane, and (b) as shown on the right of Fig. 14, the four approaching rare gas atoms form bonds of equivalent strength with one another at the nuclear configuration existing at the energy minimum of the waist mode, as the four bonds which are broken with the atoms located on the diagonal axes of the neighbouring lattices. Net destabilization of the lattice occurring in the $Q_{3}$ mode contraction arises from the weakening of the eight RG-RG bonds existing between the RG atoms in the first and second sphere of interaction on the $X-Y$ plane, represented by the black and grey circles respectively in Fig. 14. The extent of the contractions occurring in the $X-Y$ plane for the energy minimum of the waist mode in the $\mathrm{Zn} / \mathrm{Kr}$ system are drawn to scale in Fig. 14 with the lattice parameter of solid Kr. Lattice destabilization energies of 176, 130, and $624 \mathrm{~cm}^{-1}$ are calculated for the energy minimum of the $Q_{3}$ mode in the $\mathrm{Zn} / \mathrm{Ar}, \mathrm{Zn} / \mathrm{Kr}$, and $\mathrm{Zn} / \mathrm{Xe}$ systems as collected in Table III.

The emission energies resulting from $Q_{3}$, the waist vibronic mode, on the excited ${ }^{1} T_{1 u}$ state of the $\mathrm{Zn} / \mathrm{RG}$ systems can be obtained, as outlined earlier for the body mode, when the ground state energies are calculated, with Eq. 1, at the internuclear distance of the excited state energy minimum. For Ar and Kr the "waist"' mode results in emission energies of 46038 and $41634 \mathrm{~cm}^{-1}$, values which compare well with 45725 and $41753 \mathrm{~cm}^{-1}$ the 218.7- and the 239.5-nm emis-
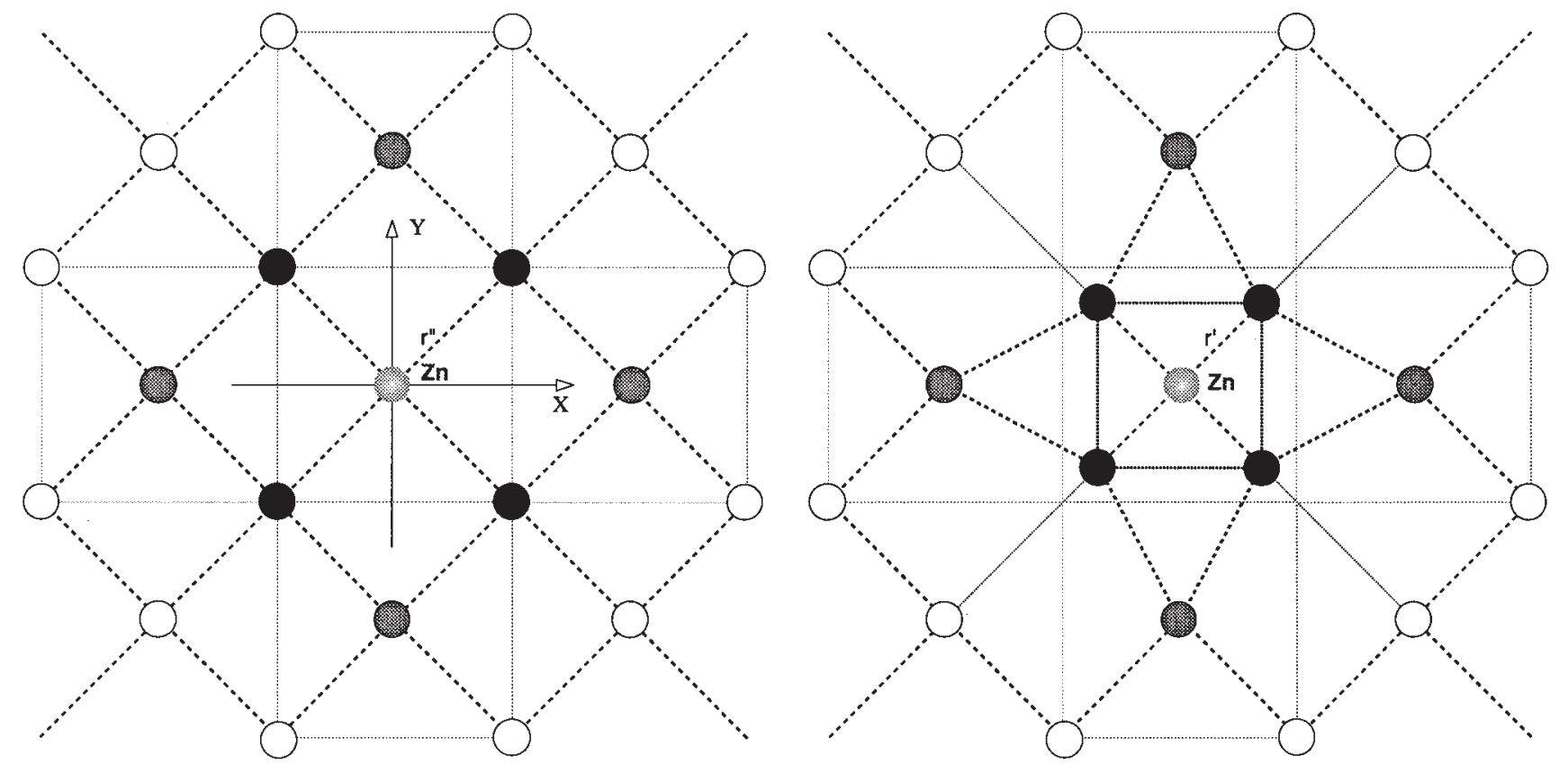

FIG. 14. Illustration of the atomic motions occurring on the $X-Y$ plane in the rare gas lattice with the "waist”' $Q_{3}$ vibronic modes of the Zn/Kr system. The locations of the four contracted $\mathrm{Kr}$ atoms at the energy minimum on the $Q_{3}$ mode of $\mathrm{Zn}\left(p_{z}\right) / \mathrm{Kr}$ are shown to scale with the lattice parameter of solid Kr. The four rare gas bonds formed and the four bonds broken in the $Q_{3}$ mode are evident in the diagram on the right. The shading used indicates the rare gas atoms in the first and second spheres of interaction around atomic $\mathrm{Zn}$. 


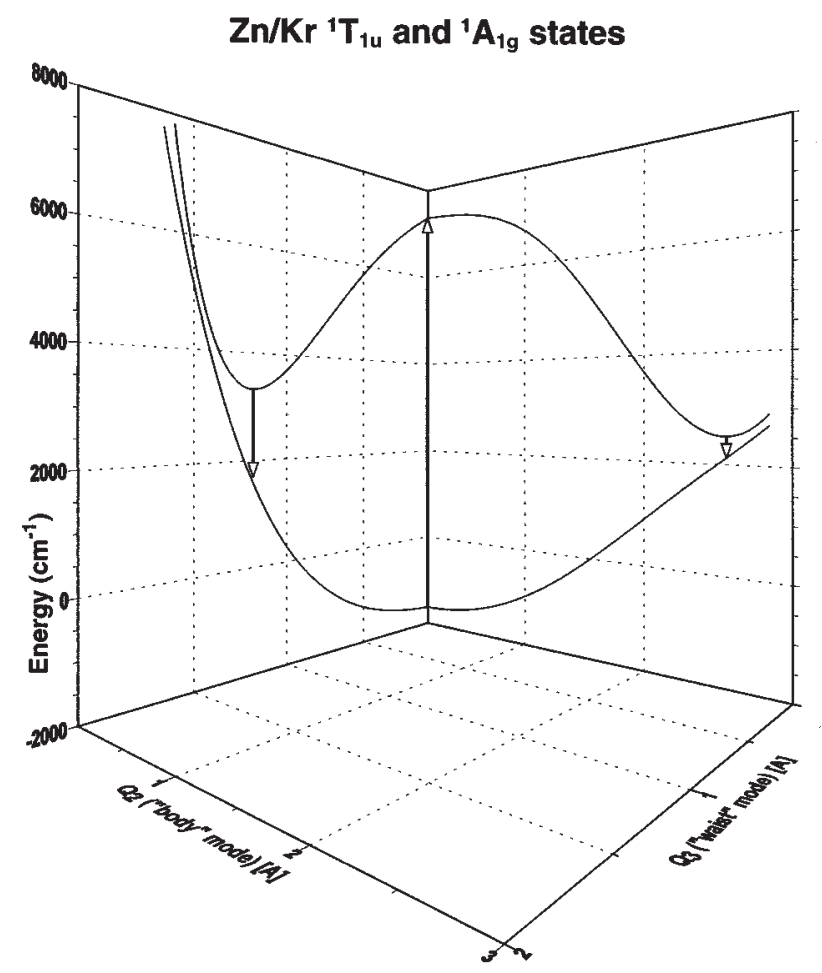

FIG. 15. Excited ${ }^{1} T_{1 u}$ and ground ${ }^{1} A_{1 g}$ electronic state energy curves arising for interaction with the "body" $Q_{2}$ and "waist" $Q_{3}$ vibronic modes of $\mathrm{Zn} / \mathrm{Kr}$. For the purposes of presentation, the excited ${ }^{1} T_{1 u}$ state energy has been lowered $40000 \mathrm{~cm}^{-1}$ from its real value. Note the very different slopes on the two vibronic modes in the Franck-Condon regions accessible in absorption.

sion bands observed in $\mathrm{Zn} / \mathrm{Ar}$ and $\mathrm{Zn} / \mathrm{Kr}$ respectively.

Depictions of the $Q_{2}$ and $Q_{3}$ vibronic interactions in the excited ${ }^{1} T_{1 u}$ and ground ${ }^{1} A_{1 g}$ electronic states of the $\mathrm{Zn} / \mathrm{Kr}$ system are shown in Fig. 15. The vertical upward facing arrow indicates the regions of the excited ${ }^{1} T_{1 u}$ state reached in absorption from the ground ${ }^{1} A_{1 g}$ state of atomic Zn located in a substitutional site. Clearly the two vibronic modes have very different gradients $\delta V / \delta Q_{n}$ in the Franck-Condon accessible regions. Thus one insight which can be gained from the present theoretical analysis of the vibronic interactions is the reason why the high energy emission can coexist in the presence of the lower energy emission. Because of the very fast rate at which relaxation occurs along $Q_{3}$ following photoexcitation, the high energy level is fed preferentially due its steeper gradient $\delta V / \delta Q_{3}$ compared to the relatively flat initial slope of $Q_{2}$ leading to the lower energy level. Experimental evidence of the difference in the feeding rates of the emission bands has been detected in the temporal decay profiles recorded in the $\mathrm{Zn} / \mathrm{Ar}$ and $\mathrm{Zn} / \mathrm{Kr}$ systems. Thus a risetime of a few hundred picoseconds exists on the lower energy 238- and 258.7-nm emission bands in $\mathrm{Zn} / \mathrm{Ar}$ and $\mathrm{Zn} /$ $\mathrm{Kr}$, respectively, at low temperatures $(<10 \mathrm{~K})$ whereas none is found on the higher energy 218.7- and 238.0-nm bands at these temperatures.
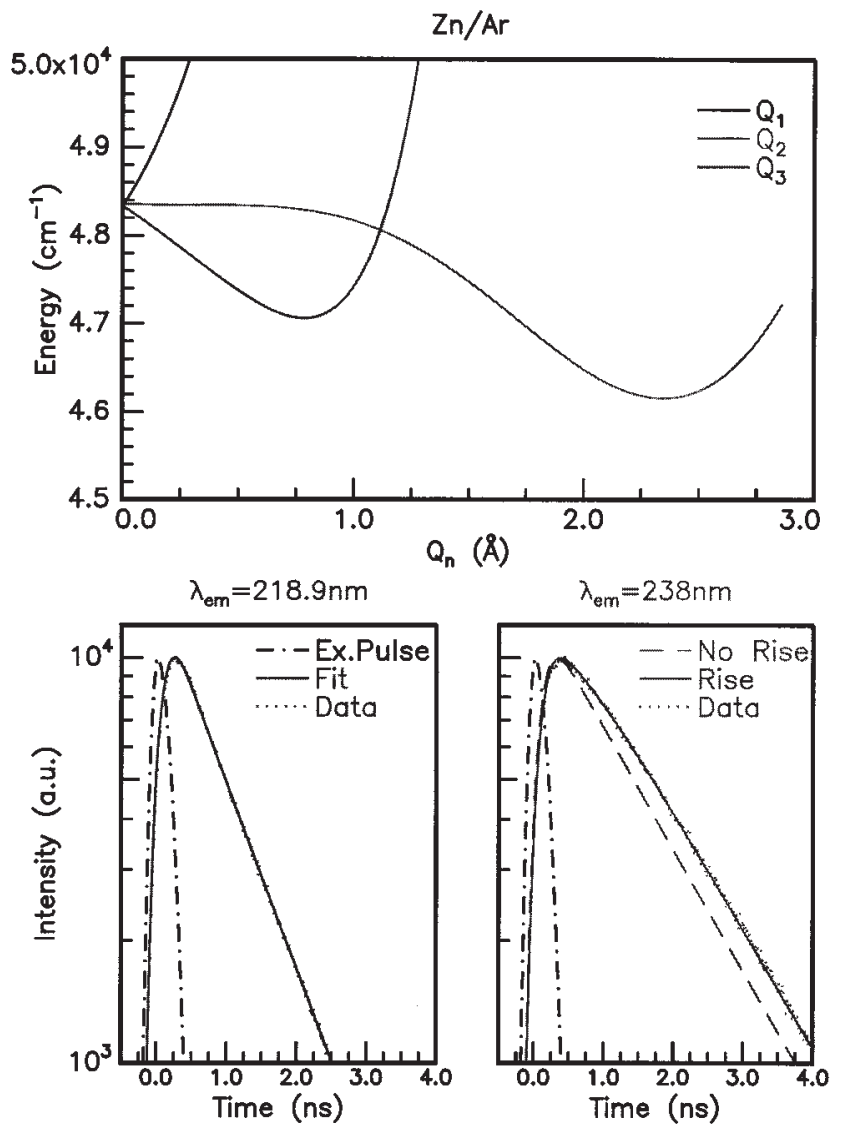

FIG. 16. A detailed comparison of the excited ${ }^{1} T_{1 u}$ electronic state energy curves in $\mathrm{Zn} / \mathrm{Ar}$ arising from interaction with the breathing, body and waist $Q_{1}, Q_{2}$, and $Q_{3}$ vibronic modes indicating the stabilization on the latter two modes and destabilization for the former. Note the very different gradients present on the stabilized $Q_{2}$, and $Q_{3}$ vibronic modes which lead to the two emission bands. The temporal profiles of the 219- and 238-nm bands of $\mathrm{Zn} / \mathrm{Ar}$ are shown underneath. The latter shows a pronounced risetime of $0.4 \mathrm{~ns}$ at $10 \mathrm{~K}$ while the $219 \mathrm{~nm}$ does not.

\section{F. Breathing mode}

A vibronic mode which can couple in both the excited and ground states of the $\mathrm{Zn} / \mathrm{RG}$ systems is the totally symmetric breathing mode, labeled $Q_{1}$. Because of the high symmetry of this vibronic mode its evaluation is straightforward as it only involves taking a simple sum over a single variable $R$ in Eqs. (1) and (2)-(4) for the ground and excited states respectively. However, calculations show that this mode does not lead to a lowering of the ${ }^{1} T_{1 u}$ state energy in $\mathrm{Zn} / \mathrm{Ar}$ and $\mathrm{Zn} / \mathrm{Kr}$ and is not expected to play a role in the observed fluorescence of the Zn/RG systems.

\section{G. Excited triplet states}

Interaction energies of the vibronic modes $Q_{2}$ and $Q_{3}$ on the spin triplet states of atomic zinc in solid rare gases $\mathrm{Zn}\left({ }^{3} T_{1 u}\right) / \mathrm{RG}$ have also been determined as analytical expressions for all the molecular triplet states of the $\mathrm{Zn} \cdot \mathrm{RG}$ diatomics exist and are collected in Table II. Calculations of the emission energies in the spin triplets of $\mathrm{Zn} / \mathrm{Ar}, \mathrm{Zn} / \mathrm{Kr}$, and $\mathrm{Zn} / \mathrm{Xe}$ were obtained following the procedure outlined above for the spin singlets and the results are summarized in 
Table III. Excellent agreement exists between the calculated (392.2 and $357.1 \mathrm{~nm}$ ) and observed (399 and $356 \mathrm{~nm}$ ) emission bands in $\mathrm{Zn} / \mathrm{Xe}$. In $\mathrm{Zn} / \mathrm{Kr}$, agreement between theory and experiment for the spin triplet is not as good as found in the spin singlets. However, the same trends are exhibited with the higher energy emission band associated with the waist mode $Q_{3}$, the lower energy band with the body mode $Q_{2}$. It will also be noticed in Table III that the energy minimum found on the spin triplets are located at nuclear configurations which involve smaller displacements from the substitutional site $(R=0)$ than in the singlets. Narrower linewidths are therefore predicted for the triplet emissions than the singlets as is indeed observed experimentally in the $\mathrm{Zn} / \mathrm{Kr}$ system.

\section{H. Singlet absorption}

The $\mathrm{Zn} / \mathrm{RG}_{18}$ calculations outlined above for emission can also be used to obtain the absorption energy, when it is remembered that this is simply the energy difference between the lower ${ }^{1} A_{1 g}$ and upper ${ }^{1} T_{1 u}$ states at $R=0$ for any of the $Q_{1}, Q_{2}$, or $Q_{3}$ vibronic modes considered. Such calculations predict transitions at 203.3, 204.1, and $212.2 \mathrm{~nm}$ for $\mathrm{Zn} / \mathrm{Ar}, \mathrm{Zn} / \mathrm{Kr}$, and $\mathrm{Zn} / \mathrm{Xe}$, respectively, all at higher energies than the component furthest to the blue in the threefold split excitation/absorption bands located at 205.4, 211.5, and 219.5 in $\mathrm{Ar}, \mathrm{Kr}$, and Xe. The reason that the absorption predictions are all to higher energy of the observed bands is that the long range attractive interactions present in the $\mathrm{Zn}\left(p_{z}\right) \cdot \mathrm{RG}$ excited $D^{1} \Sigma$ states are not being included in the repulsive-exponential functions listed for this state in Table II. This omission is most acute in the more polarizable $\mathrm{Zn} / \mathrm{Kr}$ and $\mathrm{Zn} / \mathrm{Xe}$ systems, consistent with the larger differences existing between observations and predictions in these cases. To account for the long range attractive interactions on the ${ }^{1} T_{1 u}$ state, experimentally determined $D^{1} \Sigma \mathrm{Zn} \cdot \mathrm{RG}$ potentials must be used since the $a b$ initio calculations fail to locate the weakly bound van der Waals region. Only in the case of $\mathrm{Zn} \cdot \mathrm{Xe}$ does experimental data ${ }^{21}$ exist on this weakly bound minimum $\left(134 \mathrm{~cm}^{-1}\right)$ at $5.85 \AA$. By splining the bound portion of the corresponding Morse potential to the repulsive exponential $a b$ initio $\mathrm{Zn} \cdot \mathrm{Xe}{ }^{1} \Sigma$ state curve listed in Table II, absorption at $215.7 \mathrm{~nm}$ is calculated. Significant improvement in the $\mathrm{Zn} / \mathrm{Kr}$ absorption simulation is also expected with inclusion of this long range attractive interaction on the excited $D^{1} \Sigma \mathrm{Zn}\left(p_{z}\right) \cdot \mathrm{Kr}$ state, but as mentioned above, such data are not currently available.

\section{DISCUSSION}

Of the $\mathrm{Zn}\left({ }^{1} P_{1}\right) \cdot \mathrm{RG}_{n}$ clusters considered the maximum excited state binding energy is found in the ${ }^{1} A_{1}$ state of the $\mathrm{Zn}\left({ }^{1} P_{1}\right) \cdot \mathrm{RG}_{4}$ species having a square planar structure. Unfavorable Franck-Condon factors from the ground state complex may, however, mean that the attractive region on the ${ }^{1} A_{1}$ state of the $\mathrm{Zn}\left({ }^{1} P_{1}\right) \cdot \mathrm{RG}_{4}$ species is not accessible in photoexcitation studies.

The agreement existing in Table III between the observed and predicted emission energies in the Zn/RG sys-
TABLE IV. Comparison of the site diameters of the solid rare gases and the equilibrium internuclear separations $r_{e}$ of the $\mathrm{Zn} \cdot \mathrm{RG}$ diatomics in the ground $X^{1} \Sigma_{0}$ and first excited ${ }^{1} \Pi_{1}$ electronic states. All the distances are in $\AA$ units, the lattice parameters quoted for the solid rare gases are the values at $4 \mathrm{~K}$.

\begin{tabular}{lccccc}
\hline \hline $\mathrm{RG}\urcorner$ & Lat.Parm & $I_{\mathrm{Oh}}$ site $(\varnothing)$ & Sub.site $(\varnothing)$ & $r_{e} X^{1} \Sigma_{0}$ & $r_{e}{ }^{1} \Pi_{1}$ \\
\hline $\mathrm{Ne}\urcorner$ & $4.46\urcorner$ & $1.306\urcorner$ & $3.154\urcorner$ & na $\urcorner$ & na \\
$\mathrm{Ar}\urcorner$ & $5.31\urcorner$ & $1.55\urcorner$ & $3.755\urcorner$ & $4.18\urcorner$ & 2.97 \\
$\mathrm{Kr}\urcorner$ & $5.64\urcorner$ & $1.64\urcorner$ & $3.988\urcorner$ & $4.21\urcorner$ & 2.80 \\
$\mathrm{Xe}\urcorner$ & $6.13\urcorner$ & $1.79\urcorner$ & $4.334\urcorner$ & $4.38\urcorner$ & 2.83 \\
\hline \hline
\end{tabular}

tems indicates the plausibility of using pair potentials to model the solid state spectroscopy of matrix-isolated atomic zinc. Even in the $\mathrm{Zn} / \mathrm{Xe}$ system where the $\mathrm{Zn} \cdot \mathrm{Xe}$ interactions are substantially stronger, the agreement is excellent. The poorer agreement found in the $\mathrm{Zn} / \mathrm{Ar}$ system compared to the $\mathrm{Zn} / \mathrm{Kr}$ and $\mathrm{Zn} / \mathrm{Xe}$ systems may originate in part from the enlarged site in which ground state atomic zinc resides in the Ar lattice. This can be traced back in Table IV to the ground state Zn-Ar van der Waals bond length of $4.2 \AA$ compared to a substitutional site diameter in solid Ar of only $3.75 \AA$. From the three-fold splitting evident in the $\mathrm{Zn} / \mathrm{Ar}$ excitation profile shown in Fig. 1 it is unlikely, however, that $\mathrm{Zn}$ resides in a multivacancy site in Ar. The splitting pattern on the $\mathrm{Zn} / \mathrm{Ar}$ excitation profile is indicative of a Jahn-Teller coupling in the excited for which a site of high symmetry must exist in the ground state. For neon matrices, in contrast, the excitation profiles do not show the three-fold splitting evident in all the other Zn/RG systems and, considering the trends present in Table IV, a multiple vacancy is likely in the $\mathrm{Zn} / \mathrm{Ne}$ system.

The reason for the poor agreement in the observed and simulated emission bands for the body mode of $\mathrm{Zn} / \mathrm{Ar}$ compared to the waist mode, arises from the closer approach of $\mathrm{Zn}$ to $\mathrm{Ar}$ in the body mode where the uncertainty in the repulsive $X^{1} \Sigma$ ground state potential of $\mathrm{Zn} \cdot \mathrm{Ar}$ is larger. At the excited ${ }^{1} T_{1 u}$ state energy minimum of the body mode, $\mathrm{Zn}$ in the octahedral interstitial site is located 2.67 $\AA$ away from the four nearest Ar atoms, while at the minimum of the waist mode the four $\mathrm{Ar}$ atoms are at $2.98 \AA$ from $\mathrm{Zn}$. Consequently, the energy of the ${ }^{1} A_{1 g}$ ground state of $\mathrm{Zn} / \mathrm{Ar}_{18}$ is $4129 \mathrm{~cm}^{-1}$ for the Franck-Condon accessible region of the body mode, while it is only $849 \mathrm{~cm}^{-1}$ for the waist mode as shown in Figs. 11 and 13, respectively (or Table III). Considering that a Morse function is used to describe the $X^{1} \Sigma$ ground state potential of $\mathrm{Zn}-\mathrm{Ar}$, whose bound portion only is known with accuracy, it is to be expected that the greater the extrapolation along the repulsive wall, the more unreliable the Morse description becomes. Thus we consider the use of the present $\mathrm{Zn}$-Ar ground state potential least reliable in the body mode and the likely origin of the poor agreement between observed and predicted values for this mode in $\mathrm{Zn} / \mathrm{Ar}$.

Uncertainties in the body modes of the other Zn/RG systems are less acute than in the $\mathrm{Zn} / \mathrm{Ar}$ system due to the longer range interactions involved, but none of the $\mathrm{Zn} \cdot \mathrm{RG}$ $X^{1} \Sigma_{0}$ ground state potentials can be claimed to be highly accurate. This situation arises for two reasons. First, spectro- 
scopic work on these molecules is most often obtained from laser induced fluorescence (LIF) excitation spectra, while it is for only a small number of systems ${ }^{22}$ that the resulting bound-free emission spectra have been recorded and analysed. Thus the quality of the spectroscopic data on the excited $\mathrm{Zn} \cdot \mathrm{RG}{ }^{1} \Pi_{1}$ states is very good but the situation for the $\mathrm{Zn} \cdot \mathrm{RG} X^{1} \Sigma_{0}$ ground states is not ideal. Second, Morse functions are being used in the present work to represent the repulsive regions of the $\mathrm{Zn} \cdot \mathrm{RG} X^{1} \Sigma_{0}$ state potentials, where Buckingham functions are known to provide a much more accurate description of the potential energy curves. Recording of the $\mathrm{Zn} \cdot \mathrm{RG}{ }^{1} \Pi_{1} \rightarrow X^{1} \Sigma_{0}$ bound free transitions either by spontaneous ${ }^{23}$ emission or stimulated ${ }^{24}$ laser pumping and inversion of the resulting spectral data is needed to generate accurate $X^{1} \Sigma_{0}$ state potentials and allow these calculations to be extended to do line shape simulations.

\section{CONCLUSIONS}

The existence of pairs of singlet and triplet emission bands in the $\mathrm{Zn} / \mathrm{RG}$ matrix systems is explained in terms of the two types of Jahn-Teller active vibronic modes coupling in ${ }^{1} T_{1 u}$ electronic state. The spectral positions of the emission bands are predicted accurately in the theoretical simulations. The model presented provides insight, on a microscopic level, of the atomic motions occurring in the solid in an optical cycle. Specifically, it allows identification of the high energy emission bands in the Zn/RG systems as arising from motions of the rare gas atoms surrounding the atomic zinc guest and the lower energy bands as associated with the motion of the zinc atom into an octahedral interstitial site in the lattice. It also rationalizes differences exhibited in the temporal profiles recorded for the pair of spin singlet emission bands in both the $\mathrm{Zn} / \mathrm{Ar}$ and $\mathrm{Zn} / \mathrm{Kr}$ systems.

\section{ACKNOWLEDGMENTS}

We acknowledge fruitful discussions with Benoit Soep and Bill Breckenridge. P.K. is grateful for receipt of an Irish Government, Forbairt, Science and Technology, Ph.D studentship.

\section{APPENDIX A}

Expressions for the ${ }^{1} A_{1}\left(p_{z}\right)$ and the ${ }^{1} E\left(p_{x}, p_{y}\right)$ states of the planar $\mathrm{Zn} \cdot \mathrm{RG}_{3}, \mathrm{Zn} \cdot \mathrm{RG}_{5}$, and $\mathrm{Zn} \cdot \mathrm{RG}_{6}$ cluster species in $C_{3 v}, C_{5 v}$ and $C_{6 v}$ symmetry, respectively. The values of the angles $\phi_{K}$ used in the evaluation of Eqs. (2)-(4) are also given:

$\mathrm{Zn} \cdot \mathrm{RG}_{3}, \phi_{k}=\pi / 6,5 \pi / 6$, and $3 \pi / 2$ :

$$
\begin{aligned}
& W_{A 1}(R)=3\left[\cos ^{2} \theta V_{\Sigma}(R)+\sin ^{2} \theta V_{\Pi}(R)\right], \\
& W_{E}(R)=\frac{3}{2}\left[\sin ^{2} \theta V_{\Sigma}(R)+\left(\cos ^{2} \theta+1\right) V_{\Pi}(R)\right] .
\end{aligned}
$$

$\mathrm{Zn} \cdot \mathrm{RG}_{5}, \phi_{k}=0,2 \pi / 5,4 \pi / 5,6 \pi / 5$, and $8 \pi / 5$ :

$$
W_{A 1}(R)=5\left[\cos ^{2} \theta V_{\Sigma}(R)+\sin ^{2} \theta V_{\Pi}(R)\right],
$$

$$
W_{E}(R)=\frac{5}{2}\left[\sin ^{2} \theta V_{\Sigma}(R)+\left(\cos ^{2} \theta+1\right) V_{\Pi}(R)\right] .
$$

$\mathrm{Zn} \cdot \mathrm{RG}_{6}, \phi_{k}=0, \pi / 3,2 \pi / 3, \pi, 4 \pi / 3$, and $5 \pi / 3$ :

$$
\begin{aligned}
& W_{A 1}(R)=6\left[\cos ^{2} \theta V_{\Sigma}(R)+\sin ^{2} \theta V_{\Pi}(R)\right] ; \\
& W_{E}(R)=3\left[\sin ^{2} \theta V_{\Sigma}(R)+\left(\cos ^{2} \theta+1\right) V_{\Pi}(R)\right] .
\end{aligned}
$$

\section{APPENDIX B}

The distances $r$ of each rare gas atom to the center of mass of the planar clusters are obtained by applying the general Law of cosines

$$
R_{3}^{2}=R_{2}^{2}+R_{1}^{2}-2 R_{2} R_{1} \cos \phi
$$

to the regular structures involved. In these structures $r=R_{2}$ $=R_{1}$ and using the RG-RG bond lengths $r_{e}$ for $R_{3}$ the expression above simplifies to $r^{2}=r_{e}^{2} /(2[1-\cos \phi])$. Making substitutions for the angles $\phi=2 \pi / 3, \pi / 2,2 \pi / 5$, and $\pi / 3$ for the triangular, square, pentagonal, and hexagonal structures the following values of $r$,

$$
r_{e} / \sqrt{3}, r_{e} / \sqrt{2}, r_{e} / \sqrt{2[1-\cos (2 \pi / 5)]},
$$

and $r_{e}$ are obtained for the planar $\mathrm{Zn} \cdot \mathrm{RG}_{3}, \mathrm{Zn} \cdot \mathrm{RG}_{4}$, $\mathrm{Zn} \cdot \mathrm{RG}_{5}$, and $\mathrm{Zn} \cdot \mathrm{RG}_{6}$ cluster species, respectively.

${ }^{1}$ J. G. McCaffrey and G. A. Ozin, J. Chem. Phys. 101, 10354 (1994).

${ }^{2}$ J. G. Kaup and W. H. Breckenridge, J. Phys. Chem. 99, 13701 (1995).

${ }^{3}$ V. A. Bracken, P. N. Kerins, P. Gürtler, and J. G. McCaffrey, J. Luminesc. (in press).

${ }^{4}$ Zn containing samples were also prepared from the photolysis of dimethylzinc in the solid rare gases. After annealing photolyzed DMZ/RG samples, spectra identical to those presented herein for the pure $\mathrm{Zn} / \mathrm{RG}$ depositions were obtained.

${ }^{5}$ B. Hoffmann-Millack, A. Klein, H. Lagier, B. Maid, and J. Hormes, Chem. Phys. 136, 453 (1989).

${ }^{6}$ The maximum temperatures used in annealing of the $\mathrm{Zn} / \mathrm{Ar}, \mathrm{Zn} / \mathrm{Kr}$, and $\mathrm{Zn} / \mathrm{Xe}$ solids were 33, 46, and $65 \mathrm{~K}$, respectively.

${ }^{7}$ S. Bililign, M. Gutowski, J. Simons, and W. H. Breckenridge, J. Chem. Phys. 99, 3815 (1993).

${ }^{8}$ I. Wallace, R. R. Bennett, and W. H. Breckenridge, Chem. Phys. Lett. 153, 127 (1988).

${ }^{9}$ C. E. Moore, “Atomic Energy Levels,”’ Vol. 1, National Bureau of Standards Circular Number 467 (U.S. Government Printing Office: Washington D.C., 1957), p. 125.

${ }^{10}$ J. Zuniga, A. Bastida, A. Requena, N. Halberstadt, and J. Beswick, J. Chem. Phys. 98, 1007 (1993).

${ }^{11}$ A. Bastida, J. Zuniga, A. Requena, B. Soep, N. Halberstadt, and J. Beswick, J. Chim. Phys. (Paris) 92, 384 (1995).

${ }^{12}$ S. Martrenchard-Barra, C. Jouvet, C. Lardeux-Dedonder, and D. Solgadi, J. Chem. Phys. 98, 5281 (1993).

${ }^{13}$ C. Crepin and A. Tramer, J. Chem. Phys. 97, 4772 (1992).

${ }^{14}$ W. H. Breckenridge, private communication, 1996.

${ }^{15}$ O. Roncero, J. Beswick, N. Halberstadt, and B. Soep, in Dynamics of Polyatomic van der Waals Complexes, edited by N. Halberstadt and K. Janda (Plenum Press, New York, 1990), Vol. 227, p. 471.

${ }^{16}$ R. N. Zare, Angular Momentum (Wiley-Interscience, New York, 1988).

${ }^{17}$ I. Wallace, J. Ryter, and W. H. Breckenridge, J. Chem. Phys. 96, 136 (1992).

${ }^{18}$ A. Bastida, J. Zuniga, A. Requena, N. Halberstadt, and J. Beswick, Farad. Disc., 97, 131 (1994).

${ }^{19}$ Choosing the highest symmetry approach yields the limiting energy curves of the attractive and the repulsive energy surfaces, i.e., the lowest energy for the former, the highest for the latter.

${ }^{20}$ I. B. Bersuker, The Jahn-Teller Effect and Vibronic Interactions in Modern Chemistry (Plenum, New York, 1983). 
${ }^{21}$ I. Wallace, J. G. Kaup, and W. H. Breckenridge, J. Phys. Chem. 95, 8060 (1991).

${ }^{22}$ W. H. Breckenridge, C. Jouvet, and B. Soep, "Metal-atom/rare-gas van der Waals complexes," in Advances in Metal and Semiconductor Clusters, Vol. III, edited by M. A. Duncan (JAI Press, Greenwich, 1995).
${ }^{23}$ J. G. McCaffrey, D. J. Funk, and W. H. Breckenridge, J. Chem. Phys. 100, 955 (1994).

${ }^{24} \mathrm{~L}$. Li and R. W. Field, Molecular Dynamics and Spectroscopy by Stimulated Emission Pumping, edited by H-L. Dai and R. W. Field (World Scientific, Singapore, 1995). 
Journal of Chemical Physics is copyrighted by AIP Publishing LLC (AIP). Reuse of AIP content is subject to the terms at: http://scitation.aip.org/termsconditions. For more information, see http://publishing.aip.org/authors/rights-and-permissions. 